Check for updates

Cite this: RSC Adv., 2018, 8, 38673

Received 24th September 2018 Accepted 10th November 2018

DOI: 10.1039/c8ra07920j

rsc.li/rsc-advances

\section{Synthesis of $8^{\prime}, 11^{\prime}$-dihydrospiro[cyclohexane-1,2'- oxepino[2,3-h] chromen]-4' $\left(3^{\prime} H\right)$-ones with ring closing metathesis as a key step $\dagger$}

\author{
Prathima K, (D) a Ashok D, (D) *a Sarasija M, ${ }^{\text {b }}$ Prabhakar Sripadi, ${ }^{\mathrm{c}}$ Madhu Vemula, ${ }^{\mathrm{c}}$ \\ Venkata S. Komarraju, 'Biswajit Gorai ${ }^{d}$ and Shyam Prakash ${ }^{d}$
}

A series of novel hybrid molecular entities incorporating various spiro chromanone scaffolds onto the benzannulated oxepine core moiety were synthesised using allylation, Claisen rearrangement, Kabbe condensation and Ring Closing Metathesis (RCM) as a key step. During the synthesis we found that the nitrogen functionality in the substrate influences significantly the catalyst load due to electronic effects. Several iterations have been carried out to achieve complete conversion to products $6 \mathrm{a}-6 \mathrm{e}$.

\section{Introduction}

The combination of two or more pharmacophores into a single molecule is an effective method used in medicinal chemistry to synthesize plausible lead molecules. Incorporation of drug pharmacophores into a single entity in order to enhance or amplify its mode of action is gaining popularity in medicinal chemistry. This type of combination of two or more pharmacophores either linked or fused together to create a new molecule is known as molecular hybridization and the resulting hybrid molecules may exhibit synergistic or additive biological effects. Hybrid structures, obtained from combining at least two biologically significant moieties, have emerged as a novel approach in finding new chemical entities. ${ }^{\mathbf{1 - 4}}$ They are characterized by increased biological activity. Despite considerable efforts expended in this area, it is still a challenge to develop efficient methodologies and strategies for producing such compounds with the desired substituents and functional groups. One such pharmacophoric unit is chromone which is recognized as a useful motif for the design of biologically relevant entities and is also prevalent in many natural products. ${ }^{5-10}$

Spiro chromanones too are known to be bio-active molecules with activities ranging from anti-inflammatory, anti-cancer, anti-bacterial and anti-fungal properties.

\footnotetext{
${ }^{a}$ Department of Chemistry, Osmania University, Hyderabad, India. E-mail: ashokdou@gmail.com

${ }^{b}$ Department of Chemistry, Satavahana University, Karimnagar, Telangana, India 505001

'Analytical Chemistry \& Mass Spectrometry Division, CSIR - Indian Institute of Chemical Technology, India

${ }^{d}$ Department of Laboratory Medicine, All India Institute of Medical Sciences, India

$\dagger$ Electronic supplementary information (ESI) available. See DOI: $10.1039 / \mathrm{c} 8 \mathrm{ra} 07920 \mathrm{j}$
}

Spiro-heterocycles in general have a wide range of biological properties $^{11-15}$ like spiro oxindoles have antitumor and antibiotic activity. Aza spiro cyclic compounds too are important medicinally and have been used to treat depression, anxiety and pain. ${ }^{\mathbf{1 2}}$ Likewise, oxepines are structurally important motifs in many natural products and constitute the core structure of biologically active molecules. We envisioned that combination of both these important frameworks i.e. the spiro chromanone and oxepine will provide a novel and unprecedented scaffold. Oxepines are medium sized heterocycles and can be constructed by three distinct approaches viz intramolecular cyclization, annulation and ring expansion. Apart from this they can also be constructed by Ring Closing Metathesis (RCM). ${ }^{\mathbf{1 6 - 1 8}}$ It is thought to be a powerful $\mathrm{C}=\mathrm{C}$ bond forming reaction that occurs under very mild conditions. RCM of alkenes is indispensable to the synthesis of cyclic structures. It is a highly efficient reaction for the synthesis of carbocyclic, heterocyclic and fused ring frameworks and is used extensively in the synthesis of biologically active molecules. While most RCM reactions are performed using either Grubbs first-generation (G I) or Grubbs second-generation catalyst (G II) (Fig. 1), the latter has enjoyed wide application in organic synthesis due to its high functional group tolerance. ${ }^{19}$ However, in the case of metathetic cyclization, not every ring size is accessible with the same ease. The easiest reactions are those affording 5-to 7membered rings with the entropic and enthalpic factors becoming less favourable with increasing size.

\section{Results and discussion}

2,4-Dihydroxy acetophenone is allylated using allyl bromide and $\mathrm{K}_{2} \mathrm{CO}_{3}$ in acetone and the resulting 4-O-allylated product is made to undergo Claisen rearrangement in $N, N$-diethyl aniline to obtain a free $\mathrm{OH}$ group which is again allylated to yield product 1. This on Kabbe condensation with various cyclic 
<smiles>Cl[PH](Cl)([Ge])([Ge])=Cc1ccccc1</smiles>

G I

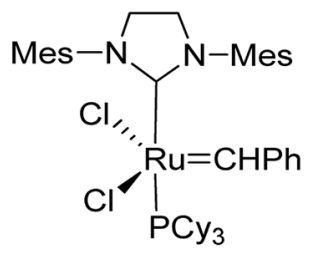

G II
Fig. 1 Grubbs catalysts

ketones gave spiro cyclic substrates (3a-3h) and with different substituted piperidones gave aza spiro cyclic substrates $(\mathbf{5 a}-\mathbf{5 j})$ (Scheme1). $\mathbf{3} \mathbf{i}$ and $\mathbf{3} \mathbf{j}$ were also produced from their respective cyclic ketones. After screening several reaction conditions, it was concluded that this condensation was best affected by using pyrrolidine as secondary amine for in situ enamine generation and ethanol/toluene as solvent at $80{ }^{\circ} \mathrm{C}$, which resulted in good yields.

Exposure of dienes $3 \mathbf{a}-\mathbf{3 h}$ to G II under our optimized conditions $\left(2 \mathrm{~mol} \%\right.$, toluene, $70{ }^{\circ} \mathrm{C}$ ) resulted in clean formation of the $\mathbf{4 a - 4 h}$ in good to excellent yields (65-91\%) within 20-30 minutes (Scheme 2). $\mathbf{4} \mathbf{i}$ and $\mathbf{4} \mathbf{j}$ were also synthesised easily from their substrates $\mathbf{3 i}$ and $\mathbf{3 j}$. Successful metathesis of abovementioned spiro cyclic substrates to give good yields of benzannulated oxepine spiro chromanones $\mathbf{4 a}-\mathbf{4 j}$ motivated us to explore the synthetic potential of G-II for our aza spiro cyclic substrates $\mathbf{5 a}-\mathbf{5 j}$ also. Thus, a series of products that could serve as precursors for the RCM was constructed in order to evaluate the scope of this reaction. In the first run, these substrates $\mathbf{5 a}-\mathbf{5 j}$ $(0.18 \mathrm{mmol}$; Table 1$)$ were stirred with G-II $(2 \mathrm{~mol} \%)$ in dry and degassed toluene at $70{ }^{\circ} \mathrm{C}$ maintaining the rest of the standard experimental protocol for metathesis reaction. Complete conversion to the products did not occur for $\mathbf{5 a - 5 e}$ (entries $1,5,8,11$ and 13; Table 1) which is verified by GC-MS (Table 1). Metathesis at higher temperature of $110{ }^{\circ} \mathrm{C}$ keeping the catalyst load same, also did not lead to full conversion, (entry 9; Table 1). Substrates having either a protecting group or an electron withdrawing group have proceeded smoothly (entries 15-19; Table 1). In short, electron deficient aza spiro cycles are proven to be efficient substrates for RCM compared to those with excess electrons. $^{20-22}$ The reason is attributed to the fact that the increased basicity on nitrogen is leading to the decomposition of catalyst, thus reducing availability at the site of action. ${ }^{23}$ When the reaction was monitored using a standard amount of 2 mol\% of G II, carbene transfer must have occurred to a large extent at the basic nitrogen, where the ruthenium metal centre might have been stabilized by chelation. Such stabilization, in turn, could have reduced the concentration of the active catalyst in the reaction resulting in a negative impact on the rate of the metathesis.

\section{Conclusion}

Sterically accessible Lewis bases are known to play an important role in the decomposition of metathesis catalysts. Many RCM reactions have been reported in case of nitrogen containing compounds where it is either protected or attached to an electron withdrawing group. But metathesis of substrates having a nitrogen functionality away from metathesis site does not seem to have been reported. We have tried to attempt RCM on substrates without protecting groups on nitrogen as in $\mathbf{5 a - 5 e}$ and studied the amount of catalyst load required for its completion. In short, we were able to achieve the synthesis of our target molecules though $\mathbf{5 a - 5 e}$ were troublesome and required multiple iterations.

\section{Experimental section}

\section{General}

Column chromatography was performed using Finar 60-120 \& 100-200 mesh silica gel and monitored using analytical thinlayer chromatography (TLC) carried out on 0.25 Merck silica gel plates (60 F-254). Visualization was by quenching of UV fluorescence $\left(\lambda_{\max }=254 \mathrm{~nm}\right)$ and by charring with freshly prepared anisaldehyde charring solution. Flash column chromatography was carried out using 60-120 mesh silica gel; samples were applied as saturated solutions in an appropriate solvent or pre-adsorbed onto the minimum quantity of silica. Except as otherwise indicated, reactions were carried out using oven-dried glassware under nitrogen with dry, freshly distilled solvents. Toluene was dried by refluxing with calcium chloride for $1 \mathrm{~h}$, distilled under nitrogen atmosphere and dried using $4 \AA$ molecular sieves. Ethanol, acetone, DMF and $N, N$-diethyl

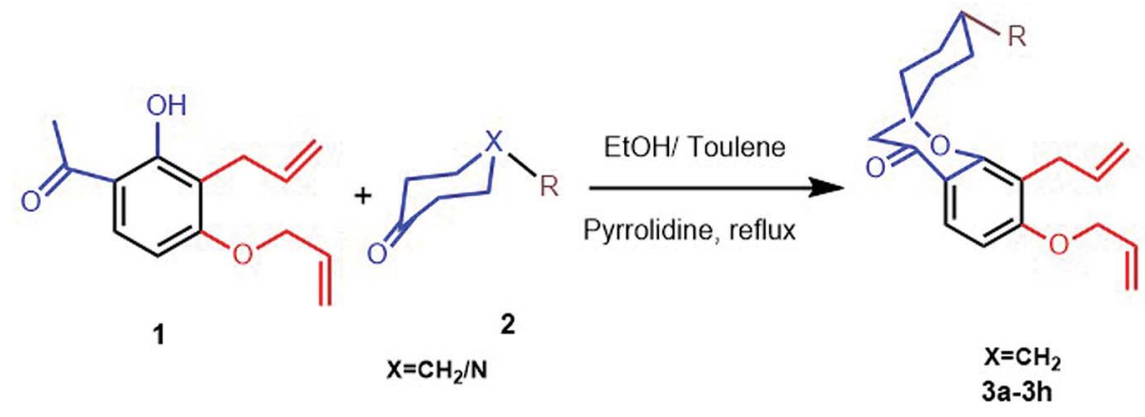

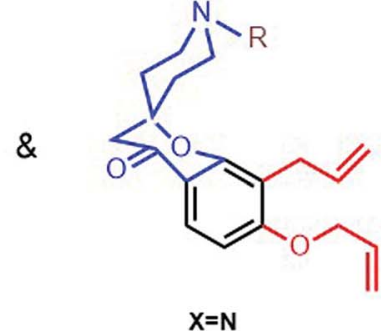

$5 a-5 j$

Scheme 1 Syntheses of metathesis substrates. 


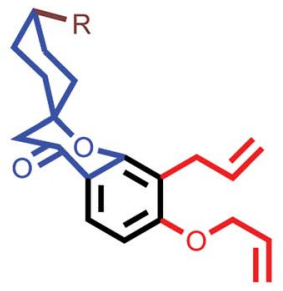

$3 a-3 h$

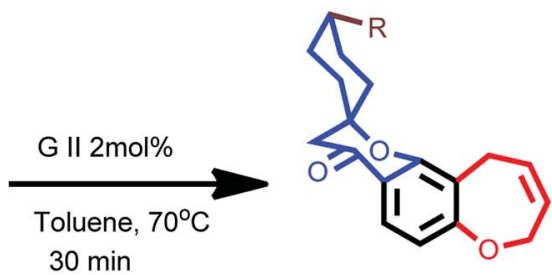

$4 a-4 h$

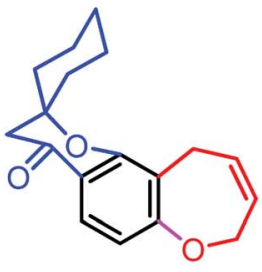

$4 a$

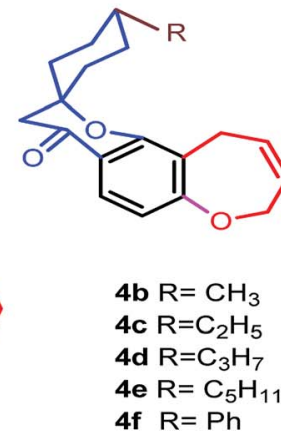

4f $\mathrm{R}=\mathrm{Ph}$

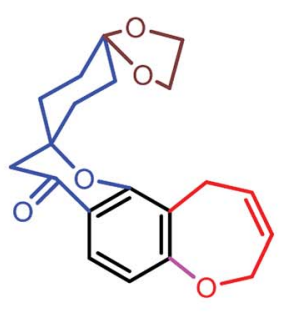

$4 \mathrm{~g}$

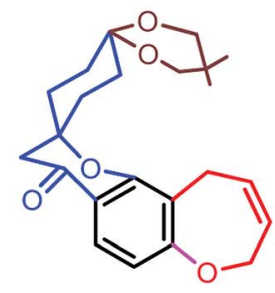

$4 \mathrm{~h}$

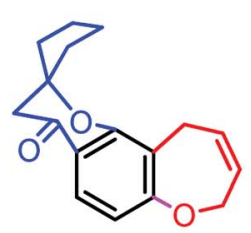

$4 \mathbf{i}$

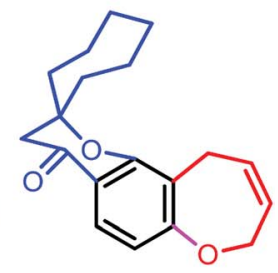

$4 \mathbf{j}$

Scheme 2 Ring closing metathesis.

aniline were purchased as laboratory grade reagents. All the other solvents were used as obtained from commercial sources. Mass spectral analysis was done on gas chromatograph - time of flight (TOF) mass spectrometer. ${ }^{1} \mathrm{H}$ NMR and ${ }^{13} \mathrm{C}$ NMR spectra were recorded on a Bruker Biospin, Avance-III Fourier Transform Digital NMR spectrometer. Nuclear magnetic resonance (NMR) spectra $\left({ }^{1} \mathrm{H}\right.$ and $\left.{ }^{13} \mathrm{C}\right)$ are recorded using either 400 or $500 \mathrm{MHz}$ instruments. Chemical shifts $(\delta)$ are referenced to the solvent signal and are quoted in ppm to the nearest $0.01 \mathrm{ppm}$. coupling constants $(J)$ are reported in Hertz to the nearest $0.1 \mathrm{~Hz}$. Multiplicities are recorded as broad peaks (brs/ brd), singlets (s), doublets (d), triplets (t), doublet of doublets of triplets (ddt), doublet of quartets (dq), doublet of heptets (dhpt), pentet of triplets (pt), triplet of doublets (td), quartet of doublets (qd), doublet of pentet (dp) and multiplets (m). Isomeric $\delta$ values are indicated by $*$. All NMR samples were prepared in deuterated chloroform and all values are quoted in ppm relative to tetra methyl silane as internal reference.

\section{Preparation of 1}

The monoallylated dihydroxy acetophenone of about 6 moles in $20 \mathrm{ml}$ of $N, N$-diethyl aniline, was refluxed for about $3-4 \mathrm{~h}$. Subsequently, the reaction mixture was cooled and diluted with ethyl acetate $(100 \mathrm{ml})$, washed thrice with $1 \mathrm{~N}$ aqueous hydrochloric acid solution $(3 \times 50 \mathrm{ml})$. The organic phase was dried with $\mathrm{Na}_{2} \mathrm{SO}_{4}$ and evaporated in vacuo. The crude mixture products were purified via column chromatography using $n$ hexane/ethyl acetate as eluent $(10: 90)$ to obtain white powdery solid in about $60-65 \%$ yield. This rearranged product is allylated once again using $\mathrm{K}_{2} \mathrm{CO}_{3}$ \& allyl bromide in acetone under reflux conditions. ${ }^{1} \mathrm{H}$ NMR $\left(\mathrm{CDCl}_{3}, 500 \mathrm{MHz}\right) \delta 12.77(\mathrm{~s}$, $1 \mathrm{H}), 7.61\left(\mathrm{~d},{ }^{3} J=9.0 \mathrm{~Hz}, 1 \mathrm{H}\right), 6.44\left(\mathrm{~d},{ }^{3} J=9.0 \mathrm{~Hz}, 1 \mathrm{H}\right), 6.02$ (ddt,
$J=15.8,10.5,5.0 \mathrm{~Hz}, 1 \mathrm{H}), 5.96(\mathrm{ddt}, J=17.8,10.5,4.8 \mathrm{~Hz}, 1 \mathrm{H})$, $5.29\left(\mathrm{dq},{ }^{3} J=10.6,2.0,{ }^{4} J=1.5 \mathrm{~Hz}, 1 \mathrm{H}\right), 5.03\left(\mathrm{dq},{ }^{3} J=17.0,2.0,{ }^{4} J\right.$ $=1.6 \mathrm{~Hz}, 1 \mathrm{H}), 4.96\left(\mathrm{dq},{ }^{3} J=9.9,2.0,{ }^{4} J=1.5 \mathrm{~Hz}, 1 \mathrm{H}\right), 4.62$ (*4.59, dt $J=5.0,1.5 \mathrm{~Hz}, 2 \mathrm{H}), 3.45$ (dt, $J=6.2,1.5 \mathrm{~Hz}, 2 \mathrm{H}), 2.56$ $\left({ }^{*} 2.59, \mathrm{~s}, 3 \mathrm{H}\right) .{ }^{13} \mathrm{C}\left(125 \mathrm{MHz}, \mathrm{CDCl}_{3}\right) \delta 221.1,180.6,180.3,154.1$, $150.9,148.7,135.8,132.9,121.5,87.2,45.0,44.5$.

\section{Preparation of $3 a-3 j \& 5 a-5 j$}

To a stirring solution of $\mathbf{1}$ in ethanol/ACN/toluene $(25 \mathrm{ml})$ was added catalytic amount of pyrrolidine $(0.05 \mathrm{mmol})$, cyclic ketones 2 in about 10-15 mmol and the resultant brown solution was stirred at $80{ }^{\circ} \mathrm{C}$ for $18 \mathrm{~h}$. After completion of the reaction, the reaction mixture was neutralised with $10 \% \mathrm{w} / \mathrm{w}$ $\mathrm{HCl}$ solution $(5 \mathrm{ml})$ and extracted with ethyl acetate $(3 \times 20$ $\mathrm{ml})$. The combined organic layers were washed with brine solution and dried over anhydrous $\mathrm{Na}_{2} \mathrm{SO}_{4}$.

3a ${ }^{1} \mathrm{H}$ NMR $\left(500 \mathrm{MHz}, \mathrm{CDCl}_{3}\right) \delta 7.74\left(\mathrm{~d},{ }^{3} J=8.7 \mathrm{~Hz}, 1 \mathrm{H}\right), 6.53$ $\left(\mathrm{d},{ }^{3} J=8.7 \mathrm{~Hz}, 1 \mathrm{H}\right), 6.03$ (ddt, $\left.J=17.7,10.3,4.8 \mathrm{~Hz}, 1 \mathrm{H}\right), 5.95$ (ddt, $J=14.2,12.8,6.4 \mathrm{~Hz}, 1 \mathrm{H}), 5.42\left(\mathrm{dq},{ }^{3} J=17.2,{ }^{4} J=1.6\right.$, $2.0 \mathrm{~Hz}, 1 \mathrm{H}), 5.28\left(\mathrm{dq},{ }^{3} J=10.6,{ }^{4} J=1.2 \mathrm{~Hz}, 1 \mathrm{H}\right), 5.07\left(\mathrm{dq},{ }^{3} J=\right.$ $\left.17.0,2.0,{ }^{4} J=1.6 \mathrm{~Hz}, 1 \mathrm{H}\right), 4.97\left(\mathrm{dq},{ }^{3} J=9.9,2.0,{ }^{4} J=1.3 \mathrm{~Hz}, 1 \mathrm{H}\right)$, $4.60(\mathrm{dt}, J=4.8,1.6 \mathrm{~Hz}, 2 \mathrm{H}), 3.45(\mathrm{dt}, J=6.4,1.3 \mathrm{~Hz}, 2 \mathrm{H}), 2.60(\mathrm{~s}$, $2 \mathrm{H}), 2.09-2.04(\mathrm{~m}, 2 \mathrm{H}), 1.72-1.64(\mathrm{~m}, 4 \mathrm{H}), 1.53-1.41(\mathrm{~m}, 4 \mathrm{H})$. ${ }^{13} \mathrm{C}$ NMR $\left(125 \mathrm{MHz}, \mathrm{CDCl}_{3}\right) \delta 210.2,180.7,176.5,154.3,151.0$, 144.3, 135.7, 134.7, 133.4, 133.1, 123.3, 98.2, 87.3, 66.5, 53.2, 48.0, 45.8, 43.5, 39.9. MS (EI) LRMS: $m / z$ 312, 269, 217 (100\%), 175, 149, 97, 69, 55.

$3 \mathbf{b}^{1} \mathrm{H}$ NMR $\left(400 \mathrm{MHz}, \mathrm{CDCl}_{3}\right) \delta 7.75\left(\mathrm{~d},{ }^{3} J=8.7 \mathrm{~Hz}, 1 \mathrm{H}\right), 6.53$ (d, $\left.{ }^{3} J=8.7 \mathrm{~Hz}, 1 \mathrm{H}\right), 6.04$ (ddt, $\left.J=17.7,10.3,4.8 \mathrm{~Hz}, 1 \mathrm{H}\right), 4$ (ddt, $J$ $=14.2,12.8,6.4 \mathrm{~Hz}, 1 \mathrm{H}), 5.42\left(\mathrm{dq},{ }^{3} J=17.2,2.0,{ }^{4} J=1.6 \mathrm{~Hz}, 1 \mathrm{H}\right)$, $5.29\left(\mathrm{dq},{ }^{3} J=10.6,2.0,{ }^{4} J=1.2 \mathrm{~Hz}, 1 \mathrm{H}\right), 5.09\left(\mathrm{dq},{ }^{3} J=17.0,2.0,{ }^{4} J\right.$ $=1.6 \mathrm{~Hz}, 1 \mathrm{H}), 5.08-4.94(\mathrm{~m}, 1 \mathrm{H}), 4.61(\mathrm{dt}, J=4.8,1.6 \mathrm{~Hz}, 2 \mathrm{H})$, 
Table 1 Optimization of reaction conditions for aza spiro chromanones

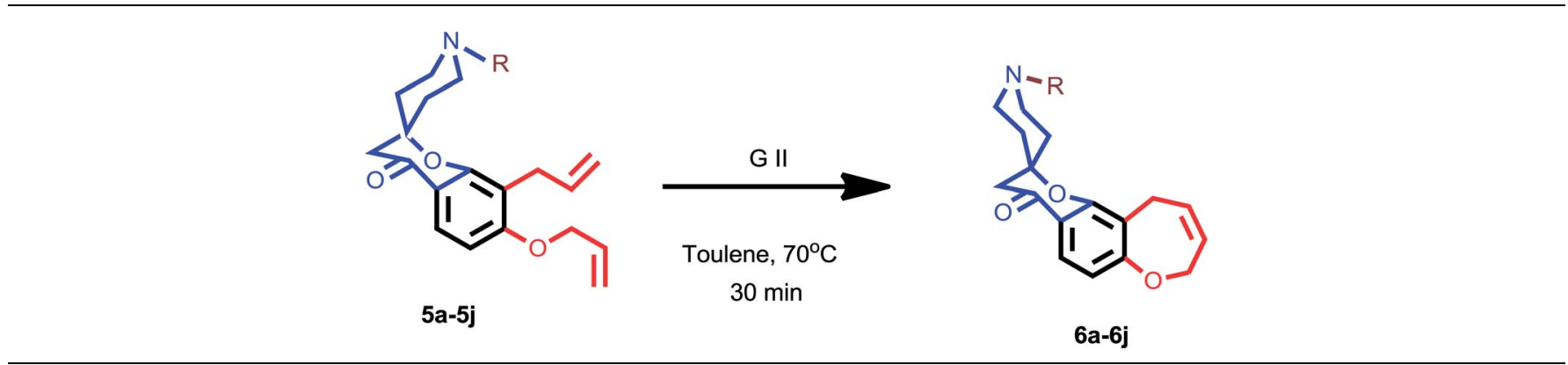<smiles>[R]NCCC12CCC(C1)Oc1ccc3c(c12)CC=CCO3</smiles>

6a R= $=\mathrm{CH}_{3}$ 6b R $=\mathrm{C}_{3} \mathrm{H}_{7}$ 6c $\mathrm{R}=\mathrm{CH}\left(\mathrm{CH}_{3}\right)_{2}$ 6d R=Bn 6e $\mathrm{R}=\mathrm{C}_{2} \mathrm{H}_{4} \mathrm{Ph}$
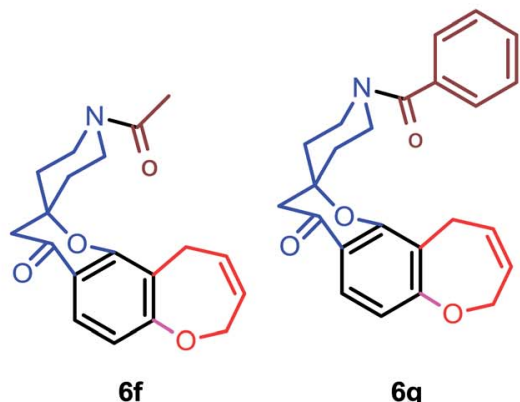

6g

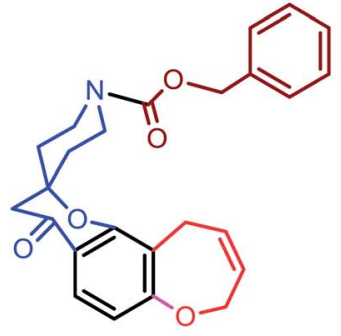

$6 \mathrm{~h}$<smiles>CCOC(=O)N1CCC2(CC1)COc1ccc3c(c12)CC=CCO3</smiles>

$6 i$<smiles>CC(C)(C)OC(=O)N1CCC2(CC1)COc1ccc3c(c12)CC=CCO3</smiles>

6j

\begin{tabular}{|c|c|c|c|c|c|c|c|}
\hline Entry & Compound & $\mathrm{R}^{a}$ & $\begin{array}{l}\text { Cat loading } \\
\text { (mol\%) }\end{array}$ & Temp & Yield $^{b}$ & Conversion & Isomer $^{d}$ ratio $(\mathbf{6 a} \mathbf{a}-\mathbf{6 j})$ \\
\hline 1 & $5 \mathbf{a}$ & $\mathrm{CH}_{3}$ & 2 & 70 & & $>53^{c}$ & \\
\hline 2 & $5 \mathbf{a}$ & $\mathrm{CH}_{3}$ & 7 & 110 & & $>95^{c}$ & \\
\hline 3 & $5 \mathbf{a}$ & $\mathrm{CH}_{3}$ & 11 & 70 & & $>95^{c}$ & \\
\hline 4 & $5 \mathbf{a}$ & $\mathrm{CH}_{3}$ & 15 & 70 & 78 & & $5: 95$ \\
\hline 5 & $5 \mathbf{b}$ & $\mathrm{C}_{3} \mathrm{H}_{7}$ & 2 & 70 & & & \\
\hline 6 & $5 \mathbf{b}$ & $\mathrm{C}_{3} \mathrm{H}_{7}$ & 3 & 70 & & $>95^{c}$ & \\
\hline 7 & $5 \mathbf{b}$ & $\mathrm{C}_{3} \mathrm{H}_{7}$ & 4 & 70 & 61 & & $20: 80$ \\
\hline 8 & $5 c$ & $\mathrm{CH}\left(\mathrm{CH}_{3}\right)_{2}$ & 2 & 70 & & $>55^{c}$ & \\
\hline 9 & $5 c$ & $\mathrm{CH}\left(\mathrm{CH}_{3}\right)_{2}$ & 2 & 110 & & $>40^{c}$ & \\
\hline 10 & $5 c$ & $\mathrm{CH}\left(\mathrm{CH}_{3}\right)_{2}$ & 3.5 & 70 & 75 & & $5: 95$ \\
\hline 11 & $5 d$ & $\mathrm{Bn}$ & 2 & 70 & & $>97^{c}$ & \\
\hline 12 & $5 d$ & $\mathrm{Bn}$ & 3 & 70 & 56 & & $2: 98$ \\
\hline 13 & $5 e$ & $\mathrm{C}_{2} \mathrm{H}_{4} \mathrm{Ph}$ & 2 & 70 & & $>95^{c}$ & \\
\hline 14 & $5 e$ & $\mathrm{C}_{2} \mathrm{H}_{4} \mathrm{Ph}$ & 4 & 70 & 85 & & $20: 80$ \\
\hline 15 & $5 f$ & COMe & 2 & 70 & 82 & & $0: 100$ \\
\hline 16 & $5 \mathrm{~g}$ & $\mathrm{Bz}$ & 2 & 70 & 87 & & $2: 98$ \\
\hline 17 & $5 h$ & $\mathrm{Cbz}$ & 2 & 70 & 89 & & $0: 100$ \\
\hline 18 & $5 i$ & $\mathrm{CO}_{2} \mathrm{Et}$ & 2 & 70 & 88 & & $20: 80$ \\
\hline 19 & $5 \mathbf{j}$ & Boc & 2 & 70 & 98 & & $20: 80$ \\
\hline
\end{tabular}

$3.46(* 3.42, \mathrm{dt}, J=6.4,1.3 \mathrm{~Hz}, 2 \mathrm{H}), 2.60(* 2.76, \mathrm{~s}, 2 \mathrm{H}), 2.12-1.91$ (m, 2H), 1.76-1.61 (m, 2H), 1.54-1.32 (m, 5H), 0.95-0.92 (m, $3 \mathrm{H}) .{ }^{13} \mathrm{C}$ NMR $\left(100 \mathrm{MHz}, \mathrm{CDCl}_{3}\right) \delta 191.9,162.4,158.1,136.0$, 132.7, 126.0, 117.4, 116.4, 115.0, 114.6, 115.1, 105.0, 104.9, 81.0, 79.2, 68.9, 48.7, 34.6, 31.6, 29.7, 27.5, 22.3. MS (EI) LRMS: $m / z$ 326, 269, 217, 175, 97, 69, 57, 44 (100\%).

$3 \mathrm{c}^{1} \mathrm{H}$ NMR (400 MHz, $\left.\mathrm{CDCl}_{3}\right) \delta 7.68\left(\mathrm{~d},{ }^{3} \mathrm{~J}=8.7 \mathrm{~Hz}, 1 \mathrm{H}\right), 6.46$ $\left(\mathrm{d},{ }^{3} J=8.7 \mathrm{~Hz}, 1 \mathrm{H}\right), 5.95(\mathrm{ddt}, J=17.7,10.3,4.8 \mathrm{~Hz}, 1 \mathrm{H}), 5.86$ (ddt, $J=14.2,12.8,6.4 \mathrm{~Hz}, 1 \mathrm{H}), 5.35\left(\mathrm{dq},{ }^{3} \mathrm{~J}=17.2,2.0,{ }^{4} \mathrm{~J}=\right.$ $1.6 \mathrm{~Hz}, 1 \mathrm{H}), 5.21\left(\mathrm{dq},{ }^{3} J=10.6,2.0,{ }^{4} J=1.2 \mathrm{~Hz}, 1 \mathrm{H}\right), 5.00\left(\mathrm{dq},{ }^{3} J\right.$ $\left.=17.0,2.0,{ }^{4} J=1.6 \mathrm{~Hz}, 1 \mathrm{H}\right), 4.94-4.87(\mathrm{~m}, 1 \mathrm{H}), 4.52(\mathrm{dt}, J=4.8$,
$1.6 \mathrm{~Hz}, 2 \mathrm{H}), 3.38\left({ }^{*} 3.35, \mathrm{dt}, J=6.4,1.3 \mathrm{~Hz}, 2 \mathrm{H}\right), 2.53(* 2.68, \mathrm{~s}$, 2H), 2.08-2.02 (*1.91-1.85 (m), m, 2H), 1.75-1.59 (m, 1H), 1.56$1.47(\mathrm{~m}, 2 \mathrm{H}), 1.36-1.18(\mathrm{~m}, 6 \mathrm{H}), 0.82(\mathrm{t}, 3 \mathrm{H}) .{ }^{13} \mathrm{C} \mathrm{NMR}(100 \mathrm{MHz}$, $\left.\mathrm{CDCl}_{3}\right) \delta 190.9,161.4,157.1,135.0,131.7,125.0,116.3,115.3$, 114.1, 113.9, 103.9, 78.5, 67.9, 47.7, 37.3, 33.5, 28.5, 26.5, 26.3, 10.4. MS (EI) LRMS: $m / z$ 340, 299, 269, 217, 175, 149, 97, 69, 57, $41(100 \%)$.

3d ${ }^{1} \mathrm{H}$ NMR (400 MHz, $\left.\mathrm{CDCl}_{3}\right) \delta 7.68\left(\mathrm{~d},{ }^{3} J=8.7 \mathrm{~Hz}, 1 \mathrm{H}\right), 6.46$ (d, ${ }^{3} J=8.7 \mathrm{~Hz}, 1 \mathrm{H}$ ), 5.97 (ddt, $\left.J=17.7,10.3,4.8 \mathrm{~Hz}, 1 \mathrm{H}\right), 5.86$ (ddt, $J=14.2,12.8,6.4 \mathrm{~Hz}, 1 \mathrm{H}), 5.34\left(\mathrm{dq},{ }^{3} J=17.2,2,{ }^{4} J=1.6 \mathrm{~Hz}\right.$, $1 \mathrm{H}), 5.21\left(\mathrm{dq},{ }^{3} J=10.6,2.0,{ }^{4} \mathrm{~J}=1.2 \mathrm{~Hz}, 1 \mathrm{H}\right), 5.00\left(\mathrm{dq},{ }^{3} J=17.0\right.$, 
$\left.2.0,{ }^{4} J=1.6 \mathrm{~Hz}, 1 \mathrm{H}\right), 4.94-4.86(\mathrm{~m}, 1 \mathrm{H}), 4.53(\mathrm{dt}, J=4.8,1.6 \mathrm{~Hz}$, $2 \mathrm{H}), 3.38(* 3.35, \mathrm{dt}, J=6.4,1.3 \mathrm{~Hz}, 2 \mathrm{H}), 2.52(* 2.68, \mathrm{~s}, 2 \mathrm{H}), 2.08-$ 2.01 (*1.89-1.84 (m), m, 2H), 1.73-1.47 (m, 2H), 1.36-1.10 (m, 9H), $0.82(\mathrm{td}, 3 \mathrm{H}) .{ }^{13} \mathrm{C}$ NMR (100 MHz, $\left.\mathrm{CDCl}_{3}\right) \delta$ 192.0, 162.4, 158.1, 136.0, 132.7, 126.0, 117.4, 116.3, 114.9, 104.9, 79.5, 68.9, 48.7, 39.2, 36.3, 34.6, 28.2, 27.8, 27.5, 27.3, 19.9, 14.3. MS (EI) LRMS: $m / z$ 354, 269, 217 (100\%), 175, 149, 91, 41 (90\%).

$3 \mathrm{e}^{1} \mathrm{H}$ NMR (400 MHz, $\left.\mathrm{CDCl}_{3}\right) \delta 7.68\left(\mathrm{~d},{ }^{3} J=8.7 \mathrm{~Hz}, 1 \mathrm{H}\right), 6.46$ $\left(\mathrm{d},{ }^{3} J=8.7 \mathrm{~Hz}, 1 \mathrm{H}\right), 5.97$ (ddt, $\left.J=17.7,10.3,4.8 \mathrm{~Hz}, 1 \mathrm{H}\right), 5.87$ (ddt, $J=14.2,12.8,6.4 \mathrm{~Hz}, 1 \mathrm{H}), 5.35\left(\mathrm{dq},{ }^{3} \mathrm{~J}=17.2,2.0,{ }^{4} \mathrm{~J}=\right.$ $1.6 \mathrm{~Hz}, 1 \mathrm{H}), 5.21\left(\mathrm{dq},{ }^{3} J=10.6,2.0,{ }^{4} J=1.2 \mathrm{~Hz}, 1 \mathrm{H}\right), 5.00\left(\mathrm{dq},{ }^{3} J\right.$ $\left.=17.0,2.0,{ }^{4} J=1.6 \mathrm{~Hz}, 1 \mathrm{H}\right), 4.94-4.86(\mathrm{~m}, 1 \mathrm{H}), 4.54(\mathrm{dt}, J=4.8$, $1.6 \mathrm{~Hz}, 2 \mathrm{H}), 3.39(* 3.35, \mathrm{dt}, J=6.4,1.3 \mathrm{~Hz}, 2 \mathrm{H}), 2.53(* 2.68, \mathrm{~s}$, $2 \mathrm{H}), 2.08-2.01\left({ }^{*} 1.89-1.84, \mathrm{~m}, 2 \mathrm{H}\right), 1.73-1.56(\mathrm{~m}, 2 \mathrm{H}), 1.37-1.10$ $(\mathrm{m}, 13 \mathrm{H}), 0.81(\mathrm{td}, 3 \mathrm{H}) .{ }^{13} \mathrm{C} \mathrm{NMR}\left(100 \mathrm{MHz}, \mathrm{CDCl}_{3}\right) \delta$ 192.0, 162.4, 158.1, 136.0, 132.7, 126.0, 117.4, 114.9, 104.9, 79.6, 68.9, 48.7, 36.9, 36.6, 34.6, 32.1, 27.8, 27.5, 26.5, 22.6, 14.0. MS (EI) LRMS: $m / z$ 382, 341, 269, 217 (100\%), 175, 149, 43, 41 (100\%).

$3 \mathrm{f}^{1} \mathrm{H}$ NMR $\left(400 \mathrm{MHz}, \mathrm{CDCl}_{3}\right) \delta 7.78\left(\mathrm{~d},{ }^{3} \mathrm{~J}=8.7 \mathrm{~Hz}, 1 \mathrm{H}\right), 7.34-$ $7.21(\mathrm{~m}, 5 \mathrm{H}), 6.56\left(\mathrm{~d},{ }^{3} \mathrm{~J}=8.7 \mathrm{~Hz}, 1 \mathrm{H}\right), 6.09-5.96(\mathrm{~m}, 2 \mathrm{H}), 5.43$ $\left(\mathrm{dq},{ }^{3} J=17.2,2.0,{ }^{4} \mathrm{~J}=1.6 \mathrm{~Hz}, 1 \mathrm{H}\right), 5.30\left(\mathrm{dq},{ }^{3} \mathrm{~J}=10.6,2.0,{ }^{4} J=\right.$ $1.2 \mathrm{~Hz}, 1 \mathrm{H}), 5.09\left(\mathrm{dq},{ }^{3} J=17.0,2.0,{ }^{4} J=1.6 \mathrm{~Hz}, 1 \mathrm{H}\right), 5.01\left(\mathrm{dq},{ }^{3} J\right.$ $\left.=9.9,2.0,{ }^{4} J=1.3 \mathrm{~Hz}, 1 \mathrm{H}\right),(\mathrm{m}, 1 \mathrm{H}), 4.62(\mathrm{dt}, J=5.0,1.5 \mathrm{~Hz}, 2 \mathrm{H})$, 3.55 (dt, $J=6.2,1.5 \mathrm{~Hz}, 2 \mathrm{H}), 2.67\left({ }^{*} 2.91, \mathrm{~s}, 2 \mathrm{H}\right), 2.57(\mathrm{tt}, 1 \mathrm{H})$, 2.31-2.13 (m, 2H), 1.97 (qd, 2H), 1.78-1.72 (m, 2H), 1.52 (dd, 2H). ${ }^{13} \mathrm{C}$ NMR $\left(100 \mathrm{MHz}, \mathrm{CDCl}_{3}\right) \delta$ 191.7, 162.5, 158.0, 146.6, 136.0, 132.7, 128.4, 126.8, 126.1, 117.4, 116.3, 115.0, 105.1, 78.8, 69.0, 48.7, 43.3, 34.9, 28.8, 27.4. MS (EI) LRMS: $m / z$ 388, 360 (98\%), 241 (100\%), 189, 91, 77.

$3 \mathrm{~g}^{1} \mathrm{H}$ NMR $\left(400 \mathrm{MHz}, \mathrm{CDCl}_{3}\right) \delta 7.73\left(\mathrm{~d},{ }^{3} J=8.5 \mathrm{~Hz}, 1 \mathrm{H}\right), 6.68$ $\left(\mathrm{d},{ }^{3} J=8.5,1 \mathrm{H}\right), 5.91$ (pt, $\left.{ }^{3} J=5.72 .0 \mathrm{~Hz}, 1 \mathrm{H}\right), 5.58$ (dhpt, $J=$ 11.2, $1.7 \mathrm{~Hz}, 1 \mathrm{H}), 4.64-4.61(\mathrm{~m}, 2 \mathrm{H}), 4.01-3.93(\mathrm{~m}, 4 \mathrm{H}), 3.59-$ $3.57(\mathrm{~m}, 2 \mathrm{H}), 2.67(\mathrm{~s}, 2 \mathrm{H}), 2.14-2.09(\mathrm{~m}, 2 \mathrm{H}), 1.91(\mathrm{td}, 2 \mathrm{H}), 1.78-$ $1.71(\mathrm{~m}, 2 \mathrm{H}), 1.64$ (brd, 2H). ${ }^{13} \mathrm{C} \mathrm{NMR}\left(125 \mathrm{MHz}, \mathrm{CDCl}_{3}\right) \delta 209.7$, 180.8, 176.2 , 154.1, 150.9, 144.4, 135.8, 133.3, 126.3, 123.5, 97.1, 87.3, 82.7, 82.5, 65.9, 50.7, 48.5, 45.8. MS (EI) LRMS: $m / z$ 330, 231, 177, 99 (100\%), 86.

$3 \mathbf{h}^{1} \mathrm{H}$ NMR $\left(400 \mathrm{MHz}, \mathrm{CDCl}_{3}\right) \delta 7.68\left(\mathrm{~d},{ }^{3} \mathrm{~J}=8.7 \mathrm{~Hz}, 1 \mathrm{H}\right), 6.47$ $\left(\mathrm{d},{ }^{3} J=8.7 \mathrm{~Hz}, 1 \mathrm{H}\right), 5.95$ (ddt, $\left.J=17.2,10.3,4.8 \mathrm{~Hz}, 1 \mathrm{H}\right), 5.85$ (ddt, $J=14.2,12.8,6.4 \mathrm{~Hz}, 1 \mathrm{H}), 5.34\left(\mathrm{dq},{ }^{3} \mathrm{~J}=17.2,2.0,{ }^{4} \mathrm{~J}=\right.$ $1.6 \mathrm{~Hz}, 1 \mathrm{H}), 5.21\left(\mathrm{dq},{ }^{3} J=10.3,2.0,{ }^{4} J=1.2 \mathrm{~Hz}, 1 \mathrm{H}\right), 4.97\left(\mathrm{dq},{ }^{3} J\right.$ $\left.=17.0,2.0,{ }^{4} \mathrm{~J}=1.6 \mathrm{~Hz}, 1 \mathrm{H}\right), 4.90\left(\mathrm{dq},{ }^{3} \mathrm{~J}=9.9,2.0,{ }^{4} \mathrm{~J}=1.3 \mathrm{~Hz}\right.$, $1 \mathrm{H}), 4.53(\mathrm{dt}, J=4.8,1.6 \mathrm{~Hz}, 2 \mathrm{H}), 3.46(\mathrm{~s}, 2 \mathrm{H}), 3.38(\mathrm{~s}, 4 \mathrm{H}), 2.56$ $(\mathrm{s}, 2 \mathrm{H}), 1.96(\mathrm{t}, 4 \mathrm{H}), 1.78-1.71(\mathrm{~m}, 2 \mathrm{H}), 1.56(\mathrm{td}, 2 \mathrm{H}), 0.89(\mathrm{~s}, 6 \mathrm{H})$. ${ }^{13} \mathrm{C}$ NMR $\left(100 \mathrm{MHz}, \mathrm{CDCl}_{3}\right) \delta 190.4,161.5,156.9,134.8,131.6$, 125.1, 116.4, 115.4, 113.9, 104.2, 95.8, 78.2, 69.1, 69.0, 67.9, 46.6, 30.0, 29.1, 26.4, 21.6. MS (EI) LRMS: $m / z$ 412, 217, 141 (100\%), 128, 69.

$3 \mathrm{i}^{1} \mathrm{H}$ NMR $\left(400 \mathrm{MHz}, \mathrm{CDCl}_{3}\right) \delta 7.76\left(\mathrm{~d},{ }^{3} \mathrm{~J}=8.7 \mathrm{~Hz}, 1 \mathrm{H}\right), 6.54$ $\left(\mathrm{d},{ }^{3} J=8.7 \mathrm{~Hz}, 1 \mathrm{H}\right), 6.03(\mathrm{ddt}, J=17.7,10.3,4.8 \mathrm{~Hz}, 1 \mathrm{H}), 5.90$ (ddt, $J=14.2,12.8,6.4 \mathrm{~Hz}, 1 \mathrm{H}), 5.42\left(\mathrm{dq},{ }^{3} J=17.2,2.0,{ }^{4} \mathrm{~J}=\right.$ $1.6 \mathrm{~Hz}, 1 \mathrm{H}), 5.29\left(\mathrm{dq},{ }^{3} \mathrm{~J}=10.6,2.0,{ }^{4} J=1.2 \mathrm{~Hz}, 1 \mathrm{H}\right), 5.01\left(\mathrm{dq},{ }^{3} J\right.$ $\left.=17.0,2.0,{ }^{4} \mathrm{~J}=1.6 \mathrm{~Hz}, 1 \mathrm{H}\right), 4.94\left(\mathrm{dq},{ }^{3} \mathrm{~J}=9.9,2.0,{ }^{4} \mathrm{~J}=1.3 \mathrm{~Hz}\right.$, $1 \mathrm{H}), 4.60(\mathrm{dt}, J=4.8,1.6 \mathrm{~Hz}, 2 \mathrm{H}), 3.39(\mathrm{dt}, J=6.4,1.3 \mathrm{~Hz}, 2 \mathrm{H})$, 2.77 (s, 2H), 2.09-2.03 (m, 2H), 1.91-1.83 (m, 2H), 1.76-1.67 (m, 2H), 1.65-1.58 (m, 2H). ${ }^{13} \mathrm{C}$ NMR (100 MHz, $\left.\mathrm{CDCl}_{3}\right) \delta$ 191.7, $162.1,159.0,136.0,132.7,126.1,117.3,116.5,115.3,114.5$,
105.1, 90.0, 68.8, 46.6, 37.4, 27.2, 23.8. MS (EI) LRMS: $m / z$ 298, 269, 217 (100\%), 175, 149, 91, 69.

$3 \mathbf{j}^{1} \mathrm{H}$ NMR (400 MHz, $\left.\mathrm{CDCl}_{3}\right) \delta 7.73\left(\mathrm{~d},{ }^{3} \mathrm{~J}=8.7 \mathrm{~Hz}, 1 \mathrm{H}\right), 6.52$ $\left(\mathrm{d},{ }^{3} J=8.7 \mathrm{~Hz}, 1 \mathrm{H}\right), 6.03(\mathrm{ddt}, J=17.7,10.3,4.8 \mathrm{~Hz}, 1 \mathrm{H}), 5.93$ (ddt, $J=14.2,12.8,6.4 \mathrm{~Hz}, 1 \mathrm{H}), 5.41\left(\mathrm{dq},{ }^{3} J=17.2,2.0,{ }^{4} \mathrm{~J}=\right.$ $1.6 \mathrm{~Hz}, 1 \mathrm{H}), 5.28\left(\mathrm{dq},{ }^{3} J=10.6,2.0,{ }^{4} \mathrm{~J}=1.2 \mathrm{~Hz}, 1 \mathrm{H}\right), 5.05(\mathrm{dq}$, $\left.{ }^{3} J=17.0,2.0,{ }^{4} J=1.6 \mathrm{~Hz}, 1 \mathrm{H}\right), 4.96\left(\mathrm{dq},{ }^{3} J=9.9,2.0,{ }^{4} J=1.3 \mathrm{~Hz}\right.$, $1 \mathrm{H}), 4.59(\mathrm{dt}, J=4.8,1.6 \mathrm{~Hz}, 2 \mathrm{H}), 3.44(\mathrm{dt}, J=6.4,1.3 \mathrm{~Hz}, 2 \mathrm{H})$, $2.66(\mathrm{~s}, 2 \mathrm{H}), 2.13-2.03(\mathrm{~m}, 2 \mathrm{H}), 1.77-1.63(\mathrm{~m}, 4 \mathrm{H}), 1.59-1.51(\mathrm{~m}$, $4 \mathrm{H}), 1.47-1.38(\mathrm{~m}, 2 \mathrm{H}) \cdot{ }^{13} \mathrm{C}$ NMR $\left(100 \mathrm{MHz}, \mathrm{CDCl}_{3}\right) \delta 191.9$, 162.3 , 158.5, 136.0, 132.7, 125.9, 117.3, 116.4, 115.1, 114.7, 104.9, 84.2, 68.9, 48.7, 38.4, 29.3, 27.4, 22.0. MS (EI) LRMS: $m / z$ 326, 269, 217 (100\%), 175, 149, 91, 55.

$5 \mathrm{a}^{1} \mathrm{H}$ NMR (400 MHz, $\left.\mathrm{CDCl}_{3}\right) \delta 7.69\left(\mathrm{~d},{ }^{3} J=8.7 \mathrm{~Hz}, 1 \mathrm{H}\right), 6.48$ $\left(\mathrm{d},{ }^{3} J=8.7 \mathrm{~Hz}, 1 \mathrm{H}\right), 5.99$ (ddt, $\left.J=14.3,10.3,5.0 \mathrm{~Hz}, 1 \mathrm{H}\right), 5.90$ (ddt, $J=21.0,9.3,3.0 \mathrm{~Hz}, 1 \mathrm{H}), 5.37\left(\mathrm{dq},{ }^{3} \mathrm{~J}=17.0,2.0,{ }^{4} \mathrm{~J}=\right.$ $1.7 \mathrm{~Hz}, 1 \mathrm{H}), 5.20\left(\mathrm{dq},{ }^{3} \mathrm{~J}=10.7,2.0,{ }^{4} J=1.2 \mathrm{~Hz}, 1 \mathrm{H}\right), 5.02\left(\mathrm{dq},{ }^{3} J\right.$ $\left.=17.0,2.0,{ }^{4} J=1.5 \mathrm{~Hz}, 1 \mathrm{H}\right), 4.93\left(\mathrm{dq},{ }^{3} \mathrm{~J}=10.0,2.0,{ }^{4} \mathrm{~J}=1.2 \mathrm{~Hz}\right.$, $1 \mathrm{H}), 4.52(\mathrm{dt}, J=5.0,1.5 \mathrm{~Hz}, 2 \mathrm{H}), 3.39(\mathrm{dt}, J=6.2,1.5 \mathrm{~Hz}, 2 \mathrm{H})$, 2.57 (s, 2H), $2.50(\mathrm{td}, 2 \mathrm{H}), 2.36(\mathrm{td}, 2 \mathrm{H}), 2.24$ (s, 3H), 1.99 (brd, 2H), 1.78-1.71 (m, 2H). ${ }^{13} \mathrm{C}$ NMR (100 MHz, $\left.\mathrm{CDCl}_{3}\right) \delta 190.2$, 161.5, 156.8, 134.8, 131.6, 125.1, 116.4, 115.3, 114.0, 104.2, 67.9, 49.8, 45.0, 33.4, 26.4. MS (EI) LRMS: $m / z$ 327, 326, 175, 110, 109, 96, $70(100 \%)$.

5b ${ }^{1} \mathrm{H}$ NMR (400 MHz, $\left.\mathrm{CDCl}_{3}\right) \delta 7.69\left(\mathrm{~d},{ }^{3} \mathrm{~J}=8.7 \mathrm{~Hz}, 1 \mathrm{H}\right), 6.48$ $\left(\mathrm{d},{ }^{3} J=8.7 \mathrm{~Hz}, 1 \mathrm{H}\right), 6.03(\mathrm{ddt}, J=14.3,10.3,5.0 \mathrm{~Hz}, 1 \mathrm{H}), 5.94$ (ddt, $J=21.0,9.3,3.0 \mathrm{~Hz}, 1 \mathrm{H}), 5.42\left(\mathrm{dq},{ }^{3} \mathrm{~J}=17.0,2.0,{ }^{4} \mathrm{~J}=\right.$ $1.7 \mathrm{~Hz}, 1 \mathrm{H}), 5.29\left(\mathrm{dq},{ }^{3} J=10.7,2.0,{ }^{4} J=1.2 \mathrm{~Hz}, 1 \mathrm{H}\right), 5.06\left(\mathrm{dq},{ }^{3} J\right.$ $\left.=17.0,2.0,{ }^{4} \mathrm{~J}=1.5 \mathrm{~Hz}, 1 \mathrm{H}\right), 4.99\left(\mathrm{dq},{ }^{3} \mathrm{~J}=10.0,2.0,{ }^{4} \mathrm{~J}=1.2 \mathrm{~Hz}\right.$, $1 \mathrm{H}), 4.60(\mathrm{dt}, J=5.0,1.5 \mathrm{~Hz}, 2 \mathrm{H}), 3.47(\mathrm{dt}, J=6.2,1.5 \mathrm{~Hz}, 2 \mathrm{H})$, $2.58(\mathrm{~s}, 2 \mathrm{H}) 2.39(\mathrm{td}, 3 \mathrm{H}), 2.25$ (*t, d, 2H), 1.96 (brd, 2H), 1.78$1.71(\mathrm{~m}, 4 \mathrm{H}), 1.42$ (sxt, 2H), 0.85 (t, 3H). ${ }^{13} \mathrm{C}$ NMR $(100 \mathrm{MHz}$, $\left.\mathrm{CDCl}_{3}\right) \delta 190.3,161.5,156.8,134.9,131.6,125.1,116.4,114.0$, 104.2, 76.8, 67.9, 59.6, 47.8, 33.4, 26.4, 19.1, 10.9. MS (EI) LRMS: $\mathrm{m} / \mathrm{z} 355,326$ (100\%), 110, 98, 70.

$5 \mathrm{c}^{1} \mathrm{H}$ NMR $\left(400 \mathrm{MHz}, \mathrm{CDCl}_{3}\right) \delta 7.76\left(\mathrm{~d},{ }^{3} \mathrm{~J}=8.7 \mathrm{~Hz}, 1 \mathrm{H}\right), 6.55$ $\left(\mathrm{d},{ }^{3} J=8.7 \mathrm{~Hz}, 1 \mathrm{H}\right), 6.03(\mathrm{ddt}, J=12.8,10.3,5.0 \mathrm{~Hz}, 1 \mathrm{H}), 5.94$ (ddt, $J=16.3,10.0,3.7 \mathrm{~Hz}, 1 \mathrm{H}), 5.42\left(\mathrm{dq},{ }^{3} J=17.0,2.0,{ }^{4} \mathrm{~J}=\right.$ $1.7 \mathrm{~Hz}, 1 \mathrm{H}), 5.29\left(\mathrm{dq},{ }^{3} J=10.7,2.0,{ }^{4} J=1.5 \mathrm{~Hz}, 1 \mathrm{H}\right), 5.06\left(\mathrm{dq},{ }^{3} J\right.$ $\left.=17.0,2,{ }^{4} \mathrm{~J}=1.5 \mathrm{~Hz}, 1 \mathrm{H}\right), 4.99\left(\mathrm{dq},{ }^{3} \mathrm{~J}=10.0,2.0,{ }^{4} \mathrm{~J}=1.2 \mathrm{~Hz}\right.$, $1 \mathrm{H}), 4.60(\mathrm{dt}, J=5.0,1.5 \mathrm{~Hz}, 2 \mathrm{H}), 3.47(\mathrm{dt}, J=6.2,1.5 \mathrm{~Hz}, 2 \mathrm{H})$, 2.77 (hpt, 1H), 2.65 (s, 2H) 2.64-2.56 (m, 4H), 2.06 (brd, 2H), 1.77-1.70 (m, 2H), 1.07 (d, 6H). ${ }^{13} \mathrm{C}$ NMR (100 MHz, $\mathrm{CDCl}_{3}$ ) $\delta 191.3,162.5,157.8,135.9,132.6,126.1,120.4,118.3,117.5$, 116.3, 114.9, 105.2, 3.9, 78.0, 69.0, 54.6, 47.7, 44.0, 34.6, 33.3, 27.9, 27.4, 24.8, 21.5, 18.3. MS (EI) LRMS: $m / z$ 355, 340 (100\%), 284, 124, 56, 41.

5d ${ }^{1} \mathrm{H}$ NMR $\left(400 \mathrm{MHz}, \mathrm{CDCl}_{3}\right) \delta 7.75\left(\mathrm{~d},{ }^{3} \mathrm{~J}=8.7 \mathrm{~Hz}, 1 \mathrm{H}\right), 7.31$ $(\mathrm{s}, 5 \mathrm{H}), 6.54\left(\mathrm{~d},{ }^{3} J=8.7 \mathrm{~Hz}, 1 \mathrm{H}\right), 6.04$ (ddt $J=17.1,10.5,5.0 \mathrm{~Hz}$, $1 \mathrm{H}$ ), 5.94 (ddt, $J=14.7,12.7,6.2 \mathrm{~Hz}, 1 \mathrm{H}), 5.40$ (dq, ${ }^{3} J=17.3,2.0$, $\left.{ }^{4} J=1.5 \mathrm{~Hz}, 1 \mathrm{H}\right), 5.28\left(\mathrm{dq},{ }^{3} J=10.0,2.0,{ }^{4} J=1.2 \mathrm{~Hz}, 1 \mathrm{H}\right), 5.08$ $\left(\mathrm{dq},{ }^{3} \mathrm{~J}=17.3,2.0,{ }^{4} \mathrm{~J}=1.5 \mathrm{~Hz}, 1 \mathrm{H}\right), 5.01\left(\mathrm{dq},{ }^{3} \mathrm{~J}=10.0,2.0,{ }^{4} \mathrm{~J}=\right.$ $1.2 \mathrm{~Hz}, 1 \mathrm{H}), 4.60$ (dt, $J=4.7,1.7 \mathrm{~Hz}, 2 \mathrm{H}), 3.55$ (s, 2H), 3.44 (d, $2 \mathrm{H}), 2.64(\mathrm{~s}, 2 \mathrm{H}) 2.63-2.60(\mathrm{~m}, 2 \mathrm{H}), 2.46(\mathrm{t}, 2 \mathrm{H}), 2.04(\mathrm{~d}, 2 \mathrm{H})$, 1.76-1.70 (m, 2H). $\left.{ }^{13} \mathrm{C} \mathrm{NMR} \mathrm{(100} \mathrm{MHz,} \mathrm{CDCl}_{3}\right) \delta 191.3,162.5$, 157.9 , 132.6, 129.2, 127.1, 128.2, 127.1, 126.1, 138.0, 117.5, 
116.4, 115.0, 105.2, 77.9, 69.0, 63.1, 48.7, 47.7, 34.5, 27.4. MS (EI) LRMS: $m / z$ 403, 402, 185, 146, 91 (100\%), 69.

5e ${ }^{1} \mathrm{H}$ NMR $\left(400 \mathrm{MHz}, \mathrm{CDCl}_{3}\right) \delta 7.78\left(\mathrm{~d},{ }^{3} J=8.7 \mathrm{~Hz}, 1 \mathrm{H}\right), 7.40$ $(\mathrm{s}, 5 \mathrm{H}), 6.56\left(\mathrm{~d},{ }^{3} J=8.7 \mathrm{~Hz}, 1 \mathrm{H}\right), 6.03(\mathrm{ddt}, J=14.1,10.5,5.0 \mathrm{~Hz}$, $1 \mathrm{H}), 5.97$ (ddt, $J=16.3,9.2,3.0 \mathrm{~Hz}, 1 \mathrm{H}), 5.42$ (dq, ${ }^{3} J=17.0,2.0$, $\left.{ }^{4} J=1.7 \mathrm{~Hz}, 1 \mathrm{H}\right), 5.31\left(\mathrm{dq},{ }^{3} J=10.7,2.0,{ }^{4} \mathrm{~J}=1.2 \mathrm{~Hz}, 1 \mathrm{H}\right), 5.06$ $\left(\mathrm{dq},{ }^{3} \mathrm{~J}=17.0,2.0,{ }^{4} \mathrm{~J}=1.5 \mathrm{~Hz}, 1 \mathrm{H}\right), 5.00\left(\mathrm{dq},{ }^{3} \mathrm{~J}=10.0,2.0,{ }^{4} \mathrm{~J}=\right.$ $1.2 \mathrm{~Hz}, 1 \mathrm{H}), 4.62(\mathrm{dt}, J=5.0,1.5 \mathrm{~Hz}, 2 \mathrm{H}), 3.49(\mathrm{dt}, J=6.2,1.5 \mathrm{~Hz}$, $2 \mathrm{H}), 2.84-2.75(\mathrm{~m}, 4 \mathrm{H}), 2.67(\mathrm{~s}, 2 \mathrm{H}) 2.54(\mathrm{t}, 2 \mathrm{H}), 2.07(\mathrm{~d}, 2 \mathrm{H})$, 1.81-1.63 (m, 4H). ${ }^{13} \mathrm{C}$ NMR (100 MHz, $\left.\mathrm{CDCl}_{3}\right) \delta 190.2,161.5$, $156.8,139.2$, 134.8, 131.6, 127.4, 127.6, 125.0, 116.4, 114.0, 115.3, 104.2, 76.7, 67.9, 59.4, 47.8, 46.7, 33.4, 32.7, 26.4. MS (EI) LRMS: $m / z$ 417, 327, 326 (100\%), 110.

$5 f^{1} \mathrm{H}$ NMR (400 MHz, $\left.\mathrm{CDCl}_{3}\right) \delta 7.71\left(\mathrm{~d},{ }^{3} \mathrm{~J}=8.7 \mathrm{~Hz}, 1 \mathrm{H}\right), 6.52$ $\left(\mathrm{d},{ }^{3} J=8.7 \mathrm{~Hz}, 1 \mathrm{H}\right), 5.99$ (ddt, $\left.J=17.1,10.5,5.0 \mathrm{~Hz}, 1 \mathrm{H}\right), 5.85$ (ddt, $J=14.7,12.7,6.2 \mathrm{~Hz}, 1 \mathrm{H}), 5.37$ (dd, $J=17.0, \mathrm{~Hz}, 1 \mathrm{H}), 5.23$ (dd, $J=10.5, \mathrm{~Hz}, 1 \mathrm{H}), 4.95-4.90(\mathrm{~m}, 2 \mathrm{H}), 4.54$ (d, 2H), 4.33 (brd, 1H) $3.60-3.52(\mathrm{~m}, 1 \mathrm{H}), 3.44(\mathrm{~d}, 1 \mathrm{H}), 3.39(\mathrm{~d}, 2 \mathrm{H}), 2.99(\mathrm{t}, 1 \mathrm{H})$, 2.59 (s, 2H), 2.03 (s, 3H), 1.57-1.47 (m, 2H). ${ }^{13} \mathrm{C}$ NMR (100 MHz, $\left.\mathrm{CDCl}_{3}\right) \delta 190.2,169.1,161.6,158.0,135.4,126.5,114.4,114.4$, 113.4, 110.0, 77.8, 47.5, 42.0, 37.1, 34.7, 34.0, 29.7, 27.2, 21.3. MS (EI) LRMS: $m / z$ 355, 217, 175, 81, 69 (100\%).

$5 \mathrm{~g}^{1} \mathrm{H}$ NMR $\left(400 \mathrm{MHz}, \mathrm{CDCl}_{3}\right) \delta 7.78\left(\mathrm{~d},{ }^{3} \mathrm{~J}=8.7 \mathrm{~Hz}, 1 \mathrm{H}\right), 7.40$ $(\mathrm{s}, 5 \mathrm{H}), 6.58\left(\mathrm{~d},{ }^{3} J=8.7 \mathrm{~Hz}, 1 \mathrm{H}\right), 6.06(\mathrm{ddt}, J=17.1,10.5,5.0 \mathrm{~Hz}$, $1 \mathrm{H}), 5.95$ (ddt, $J=15.7,10.0,3.7 \mathrm{~Hz}, 1 \mathrm{H}), 5.44$ (dq, ${ }^{3} J=17.3,2.0$, $\left.{ }^{4} J=1.5 \mathrm{~Hz}, 1 \mathrm{H}\right), 5.31\left(\mathrm{dq},{ }^{3} J=10.5,2.0,{ }^{4} J=1.5 \mathrm{~Hz}, 1 \mathrm{H}\right), 5.05-$ 4.99 (m, 2H), 4.61 (dt, $J=5.0,1.5 \mathrm{~Hz}, 2 \mathrm{H}$ ), 4.52 (brs, 1H), 3.60 (brs, $1 \mathrm{H}), 3.48(\mathrm{dt}, J=6.2,1.5 \mathrm{~Hz}, 3 \mathrm{H}), 3.28$ (brs, $1 \mathrm{H}), 2.68$ (s, 2H), 2.18 (brd, 2H), 1.80-1.67 (m, 1H), 1.61-1.39 (m, $1 \mathrm{H}) .{ }^{13} \mathrm{C}$ NMR (100 MHz, $\left.\mathrm{CDCl}_{3}\right) \delta 190.3,170.4,162.7,157.4,135.9,132.5$, 129.7, 126.8, 126.8, 126.3, 115.0, 16.2, 114.8, 105.6, 77.8, 69.1, 47.7, 27.4. MS (EI) LRMS: $m / z$ 417, 376, 217, 175, 105 (100\%), 77.

$5 \mathbf{h}^{1} \mathrm{H}$ NMR $\left(400 \mathrm{MHz}, \mathrm{CDCl}_{3}\right) \delta 7.77\left(\mathrm{~d},{ }^{3} \mathrm{~J}=8.7 \mathrm{~Hz}, 1 \mathrm{H}\right), 7.35$ $(\mathrm{s}, 5 \mathrm{H}), 6.58\left(\mathrm{~d},{ }^{3} J=8.7 \mathrm{~Hz}, 1 \mathrm{H}\right), 6.02(\mathrm{ddt}, J=17.1,10.5,5.0 \mathrm{~Hz}$, $1 \mathrm{H}), 5.91$ (ddt, $J=14.7,12.7,6.2 \mathrm{~Hz}, 1 \mathrm{H}), 5.44\left(\mathrm{dq},{ }^{3} J=17.3,2,{ }^{4} J\right.$ $=1.5 \mathrm{~Hz}, 1 \mathrm{H}), 5.39\left(\mathrm{dq},{ }^{3} \mathrm{~J}=10.5,2.0,{ }^{4} \mathrm{~J}=1.2 \mathrm{~Hz}, 1 \mathrm{H}\right), 5.13(\mathrm{~s}$, $2 \mathrm{H}), 4.99-4.96(\mathrm{~m}, 2 \mathrm{H}), 4.61$ (dt, $J=5.0,1.5 \mathrm{~Hz}, 2 \mathrm{H}), 4.00$ (brs, 2H), 3.46 (d, 2H), 3.26 (brs, 2H), 2.65 (s, 2H), 2.03 (brs, 2H), 1.61 (brs, 2H). ${ }^{13} \mathrm{C}$ NMR (100 MHz, $\left.\mathrm{CDCl}_{3}\right) \delta$ 190.6, 162.6, 157.5, 155.2, 136.6, 135.8, 132.5, 128.5, 127.9, 126.3, 117.6, 116.3, 115.0, 105.5, 69.0, 67.2, 47.8, 39.5, 29.7, 27.4. MS (EI) LRMS: $m / z$ 447, 357, 356, 312, 217, 215, 175, 92, 91 (100\%), 44, 41.

$5 \mathbf{i}^{1} \mathrm{H}$ NMR $\left(400 \mathrm{MHz}, \mathrm{CDCl}_{3}\right): \delta 7.78\left(\mathrm{~d},{ }^{3} \mathrm{~J}=8.7 \mathrm{~Hz}, 1 \mathrm{H}\right), 6.58$ (d, $\left.{ }^{3} J=8.7 \mathrm{~Hz}, 1 \mathrm{H}\right), 6.07$ (ddt, $\left.J=17.1,10.5,5.0 \mathrm{~Hz}, 1 \mathrm{H}\right), 5.93$ (ddt, $J=14.7,12.7,6.2 \mathrm{~Hz}, 1 \mathrm{H}), 5.44\left(\mathrm{dq},{ }^{3} J=17.3,2.0,{ }^{4} J=\right.$ $1.5 \mathrm{~Hz}, 1 \mathrm{H}), 5.31$ (dq, $\left.{ }^{3} J=10.5,2.0,{ }^{4} \mathrm{~J}=1.5 \mathrm{~Hz}, 1 \mathrm{H}\right), 5.04-4.97$ $(\mathrm{m}, 2 \mathrm{H}), 4.61(\mathrm{dt}, J=5.0,1.5 \mathrm{~Hz}, 2 \mathrm{H}), 4.13(\mathrm{q}, J=7.0,2 \mathrm{H}) 3.93$ (brs, $2 \mathrm{H}), 3.47(\mathrm{dt}, J=6.0,1.2 \mathrm{~Hz}, 2 \mathrm{H}), 3.25$ (brt, $2 \mathrm{H}) 2.65(\mathrm{~s}, 2 \mathrm{H})$, $2.02(\mathrm{~d}, 2 \mathrm{H}), 1.64-1.56(\mathrm{~m}, 2 \mathrm{H}), 1.24(\mathrm{t}, J=7.0,3 \mathrm{H}) .{ }^{13} \mathrm{C} \mathrm{NMR}$ $\left(100 \mathrm{MHz}, \mathrm{CDCl}_{3}\right) \delta 190.6,162.6,157.5,155.4,135.8,132.5$, 126.3, 117.6, 116.3, 115.0, 105.5, 77.8, 69.0, 61.4, 47.8, 39.3, 34.1, 29.7, 27.4, 14.6. MS (EI) LRMS: $m / z$ 385, 344, 217, 175 (100\%), 69.

$5 \mathbf{j}^{1} \mathrm{H}$ NMR (400 MHz, $\left.\mathrm{CDCl}_{3}\right) \delta 7.77\left(\mathrm{~d},{ }^{3} J=8.7 \mathrm{~Hz}, 1 \mathrm{H}\right), 6.57$ $\left(\mathrm{d},{ }^{3} J=8.7 \mathrm{~Hz}, 1 \mathrm{H}\right), 6.04$ (ddt, $\left.J=17.1,10.5,5.0 \mathrm{~Hz}, 1 \mathrm{H}\right), 5.92$ (ddt, $J=14.7,12.7,6.2 \mathrm{~Hz}, 1 \mathrm{H}), 5.42\left(\mathrm{dq},{ }^{3} J=17.3,2.0,{ }^{4} J=1.7 \mathrm{~Hz}, 1 \mathrm{H}\right)$, $5.30\left(\mathrm{dq},{ }^{3} \mathrm{~J}=10.5,2.0,{ }^{4} \mathrm{~J}=1.5 \mathrm{~Hz}, 1 \mathrm{H}\right), 5.03-4.96(\mathrm{~m}, 2 \mathrm{H}), 4.61$ (dt, $J=5.0,1.5 \mathrm{~Hz}, 2 \mathrm{H}), 3.89$ (brs, 2H), 3.47 (dt, $J=6.2,1.5 \mathrm{~Hz}$,
2H), 3.20 (brs, 2H) 2.65 (s, 2H), 2.04 (d, 2H), 1.60 (s, 2H), 1.46 (s, 9H). ${ }^{13} \mathrm{C}$ NMR $\left(100 \mathrm{MHz}, \mathrm{CDCl}_{3}\right) \delta$ 190.8, 162.6, 157.6, 154.7, 135.8, 132.5, 126.2, 117.6, 116.3, 115.0, 105.5, 79.7, 77.9, 69.0, 47.8, 28.4, 27.4. MS (EI) LRMS: $m / z 413$ 357, 312, 217, 175, 96, 57 $(100 \%)$.

\section{Ring closing metathesis $(4 a-4 j) \&(6 a-6 j)$}

To an oven dried Schlenk tube containing the substrate (0.18 $\mathrm{mmol})$ was added dry toluene $(10 \mathrm{ml})$ under inert atmosphere and purged with nitrogen gas for about $30 \mathrm{~min}$. This reaction mixture was then heated to $70^{\circ} \mathrm{C}$, on reaching the temperature, the catalyst ( $\mathrm{G} \mathrm{II}$ ) in solvent ( $2 \mathrm{ml}$ ) was then added using an oven dried syringe. Reaction was monitored by charring the TLC plate. After completion of the reaction, the reaction mass was then concentrated in vacuo to give brown oil. Purification was done using column chromatography.

4a ${ }^{1} \mathrm{H}$ NMR $\left(400 \mathrm{MHz}, \mathrm{CDCl}_{3}\right) \delta 7.72\left(* 7.67, \mathrm{~d},{ }^{3} J=8.5 \mathrm{~Hz}\right.$, $1 \mathrm{H}), 6.66\left({ }^{*} 6.58, \mathrm{~d},{ }^{3} J=8.5,1 \mathrm{H}\right), 5.92\left\{{ }^{*} 6.08(\mathrm{dt}, J=12.3,4.4 \mathrm{~Hz})\right.$, pt, $\left.{ }^{3} J=5.5,2.0 \mathrm{~Hz}, 1 \mathrm{H}\right\}, 5.58$ (dhpt, $\left.J=11.2,1.7 \mathrm{~Hz}, 1 \mathrm{H}\right), 4.65-$ $4.61\{* 4.30(\mathrm{t}, J=4.8 \mathrm{~Hz}), \mathrm{m}, 2 \mathrm{H}\}, 3.61-3.59(\mathrm{~m}, 2 \mathrm{H}), 2.64(\mathrm{~s}, 2 \mathrm{H})$, 2.02 (brd, 2H), 1.71-1.59 (m, 4H), 1.57-1.51 (m, 2H), 1.47-1.41 $(\mathrm{m}, 2 \mathrm{H}) .{ }^{13} \mathrm{C}$ NMR $\left(125 \mathrm{MHz}, \mathrm{CDCl}_{3}\right) \delta 210.4,183.7,175.3,145.8$, 144.9, 144.2 142.1, 135.6, 133.0, 98.5, 88.7, 66.5, 53.0, 48.0, 43.5, 40.7, 39.9. MS (EI) LRMS: $m / z$ 284, 241, 189 (100\%); HRMS (EI): $m / z$ calcd for $\mathrm{C}_{19} \mathrm{H}_{24} \mathrm{O}_{2}$ is 284.1776; found: 284.1775 .

4b ${ }^{1} \mathrm{H}$ NMR (400 MHz, $\left.\mathrm{CDCl}_{3}\right) \delta 7.72\left(\mathrm{~d},{ }^{3} \mathrm{~J}=8.5 \mathrm{~Hz}, 1 \mathrm{H}\right), 6.67$ (d, $\left.{ }^{3} J=8.5,1 \mathrm{H}\right), 5.90$ (pt, $\left.{ }^{3} J=5.72 .0 \mathrm{~Hz}, 1 \mathrm{H}\right), 5.58$ (dhpt, $J=$ 11.2, $1.7 \mathrm{~Hz}, 1 \mathrm{H}), 4.64-4.61(\mathrm{~m}, 2 \mathrm{H}), 3.60-3.55(\mathrm{~m}, 2 \mathrm{H}), 2.62$ $(* 2.77, \mathrm{~s}, 2 \mathrm{H}), 2.05(\mathrm{~m}, 2 \mathrm{H}), 1.74-1.66(\mathrm{~m}, 2 \mathrm{H}), 1.59-1.35(\mathrm{~m}$, $4 \mathrm{H}), 1.28-1.12(\mathrm{~m}, 1 \mathrm{H}), 0.97(\mathrm{~m}, 3 \mathrm{H}) .{ }^{13} \mathrm{C} \mathrm{NMR}\left(100 \mathrm{MHz}, \mathrm{CDCl}_{3}\right)$ $\delta$ 192.1 165.3, 156.8, 136.0, 127.5, 126.5, 125.9, 123.8, 120.6, 118.3, 117.4, 114.6, 79.5, 70.4, 48.6, 34.7, 31.5, 29.9, 25.1, 21.4, 22.4, 22.3. MS (EI) LRMS: $m / z$ 298, 255, 205, 203 (100\%), 189, 131, 69, 41; HRMS (EI): $m / z$ calcd for $\mathrm{C}_{19} \mathrm{H}_{22} \mathrm{O}_{3}$ is 298.1568; found: 298.1550 .

4c ${ }^{1} \mathrm{H}$ NMR (400 MHz, $\left.\mathrm{CDCl}_{3}\right) \delta 7.72\left(\mathrm{~d},{ }^{3} J=8.5 \mathrm{~Hz}, 1 \mathrm{H}\right), 6.66$ $\left(\mathrm{d},{ }^{3} J=8.5,1 \mathrm{H}\right), 5.90\left\{{ }^{*} 6.05\right.$ (dd, $\left.J=12.2,4.5 \mathrm{~Hz}\right), \mathrm{pt},{ }^{3} J=5.7$ $2.0 \mathrm{~Hz}, 1 \mathrm{H}\}, 5.58$ (dhpt, $J=11.2,1.7 \mathrm{~Hz}, 1 \mathrm{H}), 4.63-4.62(* 4.85-$ 4.82, m, 2H), 3.59-3.57 (m, 2H), 2.62 (*2.76, s, 2H), 2.16-2.10 (m, 2H), 1.62-1.56 (m, 2H), 1.40-1.19 (m, 7H), $0.90(\mathrm{t}, 3 \mathrm{H}) .{ }^{13} \mathrm{C}$ NMR (100 MHz, $\left.\mathrm{CDCl}_{3}\right) \delta 192.2,165.3,156.8,127.5,126.5,125.9$, 123.8, 117.4, 114.7, 79.9, 70.4, 48.6, 38.1, 34.4, 29.6, 27.4, 22.3, 11.3. MS (EI) LRMS: $m / z$ 312, 242, 241, 190, 189 (100\%), 160, 77, 41; HRMS (EI): $m / z$ calcd for $\mathrm{C}_{20} \mathrm{H}_{24} \mathrm{O}_{3}$ is 312.1725; found: 312.1695.

4d ${ }^{1} \mathrm{H}$ NMR (400 MHz, $\left.\mathrm{CDCl}_{3}\right) \delta 7.72\left(\mathrm{~d},{ }^{3} \mathrm{~J}=8.5 \mathrm{~Hz}, 1 \mathrm{H}\right), 6.66$ (d, $\left.{ }^{3} J=8.5,1 \mathrm{H}\right), 5.91$ (pt, ${ }^{3} J=5.72 .0 \mathrm{~Hz}, 1 \mathrm{H}$ ), 5.58 (dhpt, $J=$ 11.2, $1.7 \mathrm{~Hz}, 1 \mathrm{H}), 4.64-4.61(\mathrm{~m}, 2 \mathrm{H}), 3.60-3.55(\mathrm{~m}, 2 \mathrm{H}), 2.62$ (*2.76, s, 2H), 2.10 (brd, 2H), 1.62-1.57 (m, 3H), 1.37-1.22 (m, $8 \mathrm{H}), 0.90(\mathrm{~m}, 3 \mathrm{H}) .{ }^{13} \mathrm{C} \mathrm{NMR}\left(100 \mathrm{MHz}, \mathrm{CDCl}_{3}\right) \delta$ 192.1, 165.3, 156.8, 127.5, 126.6, 125.9, 123.8, 117.4, 114.7, 79.9, 70.4, 48.6, 39.4, 36.1, 34.4, 27.9, 22.3, 20.3, 19.9, 14.3. MS (EI) LRMS: $m / z$ 326, 255, 241 (99\%), 190, 189 (100\%), 149, 69, 57, 55, 43, 41; HRMS (EI): $m / z$ calcd for $\mathrm{C}_{21} \mathrm{H}_{26} \mathrm{O}_{3}$ is 326.1881 ; found: 326.1863 .

$4 \mathrm{e}^{1} \mathrm{H}$ NMR (400 MHz, $\left.\mathrm{CDCl}_{3}\right) \delta 7.72\left(\mathrm{~d},{ }^{3} J=8.5 \mathrm{~Hz}, 1 \mathrm{H}\right), 6.58$ (d, $\left.{ }^{3} J=8.5,1 \mathrm{H}\right), 5.94$ (pt, ${ }^{3} J=5.72 .0 \mathrm{~Hz}, 1 \mathrm{H}$ ), 5.58 (dhpt, $J=$ 
11.2, $1.7 \mathrm{~Hz}, 1 \mathrm{H}), 4.64-4.61(\mathrm{~m}, 2 \mathrm{H}), 3.60-3.55(\mathrm{~m}, 2 \mathrm{H}), 2.61$ (*2.76, s, 2H), 2.10 (brd, 2H), 1.62-1.58 (m, 2H), 1.36-1.22 (m, $13 \mathrm{H}), 0.89(\mathrm{~m}, 3 \mathrm{H}) .{ }^{13} \mathrm{C}$ NMR (100 MHz, $\left.\mathrm{CDCl}_{3}\right) \delta$ 192.1, 165.3, 156.8, 127.5, 126.6, 125.9, 123.8, 117.4, 114.7, 79.9, 70.4, 48.6, 37.0, 36.4, 34.4, 32.1, 27.9, 26.5, 22.3, 14.0. MS (EI) LRMS: $m / z$ 354, 283, 241 (100\%), 231, 190, 189 (100\%), 69, 55, 41; HRMS (EI): $m / z$ calcd for $\mathrm{C}_{23} \mathrm{H}_{30} \mathrm{O}_{3}$ is 354.2194; found: 354.2200 .

4f ${ }^{1} \mathrm{H}$ NMR $\left(400 \mathrm{MHz}, \mathrm{CDCl}_{3}\right) \delta 7.75\left(\mathrm{~d},{ }^{3} \mathrm{~J}=8.5 \mathrm{~Hz}, 1 \mathrm{H}\right), 7.35-$ $7.29(\mathrm{~m}, 2 \mathrm{H}), 7.24-7.20(\mathrm{~m}, 3 \mathrm{H}), 6.69\left(\mathrm{~d},{ }^{3} J=8.5,1 \mathrm{H}\right), 5.96\left({ }^{*} 6.13\right.$ (dd, $J=12.2,4.5 \mathrm{~Hz}$ ), pt, $\left.{ }^{3} J=5.7,2.0 \mathrm{~Hz}, 1 \mathrm{H}\right), 5.58$ (dhpt, $J=$ 11.2, $1.7 \mathrm{~Hz}, 1 \mathrm{H}), 4.65-4.62(\mathrm{~m}, 2 \mathrm{H}), 3.70-3.68$ (*3.59-3.56 (m), 2H), $2.69(* 2.91, \mathrm{~s}, 2 \mathrm{H}), 2.59(\mathrm{tt}, J=12.5,11.2,4.0 \mathrm{~Hz}, 1 \mathrm{H}), 2.24$ (brd, 2H), 1.98-1.84 (m, 2H), 1.82-1.71 (m, 2H), 1.54 (td, 2H). ${ }^{13} \mathrm{C}$ NMR $\left(100 \mathrm{MHz} \mathrm{CDCl}_{3}\right) \delta 192.0,165.4,156.7,146.5,128.5$, 127.7, 126.7, 126.0, 114.9, 79.2, 70.4, 48.5, 43.1, 34.7, 29.8, 28.8, 22.4. MS (EI) LRMS: $m / z$ 360, 286, 241, 229, 189, 177, 149, 91, 86, 84, 69, 57, 49, 41; HRMS (EI): $m / z$ calcd for $\mathrm{C}_{24} \mathrm{H}_{24} \mathrm{O}_{3}$ is 360.1725 ; found: 360.1700 .

$4 \mathrm{~g}^{1} \mathrm{H}$ NMR $\left(400 \mathrm{MHz}, \mathrm{CDCl}_{3}\right) \delta 7.73\left(\mathrm{~d},{ }^{3} \mathrm{~J}=8.5 \mathrm{~Hz}, 1 \mathrm{H}\right), 6.68$ $\left(\mathrm{d},{ }^{3} J=8.5,1 \mathrm{H}\right), 5.91\left(\mathrm{pt},{ }^{3} J=5.72 .0 \mathrm{~Hz}, 1 \mathrm{H}\right), 5.58$ (dhpt, $J=$ 11.2, $1.7 \mathrm{~Hz}, 1 \mathrm{H}), 4.64-4.61(\mathrm{~m}, 2 \mathrm{H}), 4.01-3.93$ (m, 4H), 3.59$3.57(\mathrm{~m}, 2 \mathrm{H}), 2.67$ (s, 2H), 2.14-2.09 (m, 2H), $1.91(\mathrm{td}, 2 \mathrm{H}), 1.78-$ $1.71(\mathrm{~m}, 2 \mathrm{H}), 1.64$ (brd, 2H). ${ }^{13} \mathrm{C} \mathrm{NMR}\left(100 \mathrm{MHz}, \mathrm{CDCl}_{3}\right) \delta 191.5$, 156.6, 127.4, 126.5, 126.0, 123.8, 117.2, 115.0, 107.9, 79.1, 70.4, 64.4, 64.3, 47.5, 32.2, 30.2, 22.3. MS (EI) LRMS: $m / z$ 342, 269, 217, 189 (100\%), 175, 99, 86, 84; HRMS (EI): $\mathrm{m} / \mathrm{z}$ calcd for $\mathrm{C}_{20} \mathrm{H}_{22} \mathrm{O}_{5}$ is 342.1467; found: 342.1462 .

4h ${ }^{1} \mathrm{H}$ NMR $\left(400 \mathrm{MHz}, \mathrm{CDCl}_{3}\right) \delta 7.73\left(\mathrm{~d},{ }^{3} \mathrm{~J}=8.5 \mathrm{~Hz}, 1 \mathrm{H}\right), 6.68$ $\left(\mathrm{d},{ }^{3} J=8.5,1 \mathrm{H}\right), 5.91$ (pt, $\left.{ }^{3} J=5.72 .0 \mathrm{~Hz}, 1 \mathrm{H}\right), 5.56$ (dhpt, $J=$ 11.2, $1.7 \mathrm{~Hz}, 1 \mathrm{H}), 4.64-4.61(\mathrm{~m}, 2 \mathrm{H}), 3.58-3.57(\mathrm{~m}, 2 \mathrm{H}), 3.54(\mathrm{~s}$, $2 \mathrm{H}), 3.46(\mathrm{~s}, 2 \mathrm{H}), 2.65(\mathrm{~s}, 2 \mathrm{H}), 2.11-1.99(\mathrm{~m}, 4 \mathrm{H}), 1.77-1.70(\mathrm{~m}$, 2H), 1.66-1.61 (m, 2H), 0.97 (s, 6H). $\left.{ }^{13} \mathrm{C} \mathrm{NMR} \mathrm{(100} \mathrm{MHz,} \mathrm{CDCl}_{3}\right)$ $\delta 191.5,165.4,156.7,127.4,126.6,125.9,123.8,117.2,114.9$, 96.7, 79.5, 70.1, 47.6, 30.9, 30.2, 27.6, 22.4. MS (EI) LRMS: $m / z$ 384, 298, 189, 141 (100\%), 128, 84, 69; HRMS (EI): $m / z$ calcd for $\mathrm{C}_{23} \mathrm{H}_{28} \mathrm{O}_{5}$ is 384.1936; found: 384.1933 .

$4 \mathrm{i}^{1} \mathrm{H}$ NMR $\left(400 \mathrm{MHz}, \mathrm{CDCl}_{3}\right) \delta 7.65\left(* 7.68, \mathrm{~d},{ }^{3} J=8.5 \mathrm{~Hz}\right.$, $1 \mathrm{H}), 6.58\left({ }^{*} \mathrm{~d},{ }^{3} J=8.5,1 \mathrm{H}\right), 5.81\left\{{ }^{*} 5.95(\mathrm{dt}, J=12.2,3.7 \mathrm{~Hz}), \mathrm{pt},{ }^{3} J\right.$ $=5.5,2.0 \mathrm{~Hz}, 1 \mathrm{H}\}, 5.50\left\{{ }^{*} 4.77-4.74(\mathrm{~m}), \mathrm{dhpt}, J=11.2,1.7 \mathrm{~Hz}\right.$, $1 \mathrm{H}\}, 4.56-4.52\{* 4.21(\mathrm{t}, J=4.7 \mathrm{~Hz}), \mathrm{m}, 2 \mathrm{H}\}, 3.46-3.43\{* 4.12(\mathrm{dd}$, $J=12.5,1.7 \mathrm{~Hz}), \mathrm{m}, 2 \mathrm{H}\}, 2.71(\mathrm{~s}, 2 \mathrm{H}), 2.03-1.97$ (m, 2H), 1.83$1.73(\mathrm{~m}, 2 \mathrm{H}), 1.71-1.63(\mathrm{~m}, 2 \mathrm{H}), 1.60-1.51(\mathrm{~m}, 2 \mathrm{H}) .{ }^{13} \mathrm{C} \mathrm{NMR}$ $\left(100 \mathrm{MHz}, \mathrm{CDCl}_{3}\right) \delta 192.3(* 191.5), 165.7(* 165.2), 159.8,157.9$, 130.7, 127.7 (*127.4), 126.7 (*126.1), 125.6, 124.0, 114.8, 113.6, 91.2 (*90.3), 82.5, 70.4 (*70.5), 46.4 (*46.2), 37.8, 37.4, 37.1, 23.8, 22.3. MS (EI) LRMS: $m / z$ 270, 241, 189 (100\%); HRMS (EI): $\mathrm{m} / z$ calcd for $\mathrm{C}_{17} \mathrm{H}_{18} \mathrm{O}_{3}$ is 270.1255 ; found: 270.1250 .

$4 \mathbf{j}^{1} \mathrm{H}$ NMR (400 MHz, $\left.\mathrm{CDCl}_{3}\right) \delta 7.71\left(\mathrm{~d},{ }^{3} J=8.5 \mathrm{~Hz}, 1 \mathrm{H}\right), 6.65$ $\left(\mathrm{d},{ }^{3} J=8.5,1 \mathrm{H}\right), 5.91\left(\mathrm{pt},{ }^{3} J=5.5,2.0 \mathrm{~Hz}, 1 \mathrm{H}\right), 5.58(\mathrm{dhpt}, J=$ 11.2, $1.7 \mathrm{~Hz}, 1 \mathrm{H}), 4.64-4.61(\mathrm{~m}, 2 \mathrm{H}), 3.60-3.58(\mathrm{~m}, 2 \mathrm{H}), 2.68(\mathrm{~s}$, $2 \mathrm{H}), 2.43-2.40(\mathrm{~m}, 1 \mathrm{H}), 2.12-2.07(\mathrm{~m}, 2 \mathrm{H}), 1.91-1.85(\mathrm{~m}, 1 \mathrm{H})$, 1.76-1.63 (m, 3H), 1.61-1.52 (m, 3H), 1.49-1.40 (m, 2H). ${ }^{13} \mathrm{C}$ NMR (100 MHz, $\left.\mathrm{CDCl}_{3}\right) \delta 192.1,165.3,157.2,127.4,126.6,125.8$, 123.6, 117.3 , 114.5, 84.3, 70.4, 48.7, 38.2, 28.9, 22.5, 22.0. MS (EI) LRMS: $m / z$ 298, 255, 241, 189 (100\%), 134, 98, 84, 55. HRMS (EI): $\mathrm{m} / \mathrm{z}$ calcd for $\mathrm{C}_{19} \mathrm{H}_{22} \mathrm{O}_{3}$ is 298.1568; found: 298.1558 . 6a ${ }^{1} \mathrm{H}$ NMR (400 MHz, $\left.\mathrm{CDCl}_{3}\right) \delta 7.73\left(* 7.69, \mathrm{~d},{ }^{3} J=8.5 \mathrm{~Hz}\right.$, $1 \mathrm{H}), 6.70\left({ }^{*} 6.62, \mathrm{~d},{ }^{3} J=8.5 \mathrm{~Hz}, 1 \mathrm{H}\right), 5.92\left\{\mathrm{pt},{ }^{3} J=5.5\right.$, $2.2 \mathrm{~Hz},{ }^{*} 6.10\left(\mathrm{dt},{ }^{3} J=12.0,4.5 \mathrm{~Hz}\right),{ }^{*} 6.39(\mathrm{dt}, J=7.2,1.7 \mathrm{~Hz}, 1 \mathrm{H}\}$, $5.59\left\{\mathrm{dhpt},{ }^{3} \mathrm{~J}=11.2,1.7 \mathrm{~Hz}, * 4.90\left(\mathrm{dt},{ }^{3} J=7.5,4.5 \mathrm{~Hz}\right),{ }^{*} 5.05-\right.$ $5.02(\mathrm{~m}), 1 \mathrm{H}\}, 4.65-4.62\left\{\mathrm{~m}, * 4.30\left(\mathrm{t},{ }^{3} \mathrm{~J}=4.7 \mathrm{~Hz}\right), 2 \mathrm{H}\right\}, 3.61-3.59$ $\left\{\mathrm{m},{ }^{*} 3.29-3.27(\mathrm{~m}),{ }^{*} 3.17-3.14(\mathrm{~m}), 2 \mathrm{H}\right\}, 2.68(\mathrm{~s}, 4 \mathrm{H}), 2.38(\mathrm{~s}, 4 \mathrm{H})$, 2.10-1.98 (m, 3H), 1.75-1.79 (m, 2H). ${ }^{13} \mathrm{C}$ NMR (100 MHz, $\left.\mathrm{CDCl}_{3}\right) \delta 191.3,165.4,127.6,126.4,126.0,123.8,117.37,115.0$, 70.4, 51.0, 46.1, 34.2, 29.6, 26.3, 22.4. MS (EI) LRMS: $m / z$ 299, 110, 96, 81, 70, 69 (100\%), 57, 55. HRMS (EI): $\mathrm{m} / \mathrm{z}$ calcd for $\mathrm{C}_{18} \mathrm{H}_{21} \mathrm{NO}_{3}$ is 299.1521; found: 299.1519 .

6b ${ }^{1} \mathrm{H}$ NMR (400 MHz, $\left.\mathrm{CDCl}_{3}\right) \delta 7.73\left(* 7.68, \mathrm{~d},{ }^{3} J=8.5 \mathrm{~Hz}\right.$, $1 \mathrm{H}), 6.68\left(* 6.60, \mathrm{~d},{ }^{3} J=8.5 \mathrm{~Hz}, 1 \mathrm{H}\right), 5.92\left\{\mathrm{pt},{ }^{3} J=5.5\right.$, $\left.2.0 \mathrm{~Hz},{ }^{*} 6.09\left(\mathrm{dt},{ }^{3} \mathrm{~J}=12.0,4.5 \mathrm{~Hz}\right), 1 \mathrm{H}\right\}, 5.58\left\{\mathrm{dhpt},{ }^{3} \mathrm{~J}=11.2\right.$, $\left.1.7 \mathrm{~Hz},{ }^{*} 5.45-5.39(\mathrm{~m}), 1 \mathrm{H}\right\}, 4.66-4.61\left\{\mathrm{~m}, * 4.30\left(\mathrm{t},{ }^{3} J=4.7 \mathrm{~Hz}\right)\right.$, $2 \mathrm{H}\}, 3.61-3.59(\mathrm{~m}, 2 \mathrm{H}), 2.72-2.69(\mathrm{~m}, 2 \mathrm{H}), 2.67$ (s, 2H), 2.39$2.28(\mathrm{~m}, 4 \mathrm{H}), 2.04$ (d, 2H), 1.79-1.71 (m, 2H), 1.54 (sxt, $J=7.5$, 2H), $0.91(\mathrm{t}, 3 \mathrm{H}) .{ }^{13} \mathrm{C}$ NMR (100 MHz, $\left.\mathrm{CDCl}_{3}\right)$ 191.4, 165.4, 156.5, 127.5, 126.5, 126.0, 123.8, 117.4, 115.0, 78.2, 70.4, 60.7, 48.9, 34.2, 22.5, 20.1, 11.9. MS (EI) LRMS: $m / z$ 327, 299, 198 (100\%), 187, 110, 70. HRMS (EI): $m / z$ calcd for $\mathrm{C}_{20} \mathrm{H}_{25} \mathrm{NO}_{3}$ is 327.1834; found: 327.1814 .

6c ${ }^{1} \mathrm{H}$ NMR $\left(400 \mathrm{MHz}, \mathrm{CDCl}_{3}\right) \delta 7.73\left(* 7.68, \mathrm{~d},{ }^{3} J=8.5 \mathrm{~Hz}\right.$, $1 \mathrm{H}), 6.68\left(* 6.62, \mathrm{~d},{ }^{3} J=8.5 \mathrm{~Hz}, 1 \mathrm{H}\right), 5.91\{\mathrm{pt}, J=5.7,2.0 \mathrm{~Hz}$, *6.09 (dt, $\left.\left.{ }^{3} J=12.2,4.5 \mathrm{HZ}\right), 1 \mathrm{H}\right\}, 5.58$ (dhpt, ${ }^{3} J=11.2,1.7 \mathrm{~Hz}$, $1 \mathrm{H}), 4.64-4.62\left\{\mathrm{~m}, * 4.30\left(\mathrm{t},{ }^{3} J=5.0 \mathrm{~Hz}\right), 2 \mathrm{H}\right\}, 3.63-3.59(\mathrm{~m}, 2 \mathrm{H})$, 2.76 (hpt, 1H), 2.70-2.68 (m, 1H), 2.66 (s, 2H), 2.66-2.63 (m, 1H), 2.55 (td, 2H), 2.05 (brd, 2H), 1.80-1.74 (m, 2H), 1.07 (d, 6H). ${ }^{13} \mathrm{C}$ $\mathrm{NMR}\left(100 \mathrm{MHz}, \mathrm{CDCl}_{3}\right) \delta 191.5,165.4,156.5,127.6,126.4,126.0$, 123.8, 117.4, 115.0, 78.4, 70.4, 54.5, 47.7, 44.0, 34.5, 22.4, 18.2. MS (EI) LRMS: $m / z$ 327, 313, 236, 98, 83, 71, 69, 57, 44 (100\%). HRMS (EI): $m / z$ calcd for $\mathrm{C}_{20} \mathrm{H}_{25} \mathrm{NO}_{3}$ is 327.1834; found: 327.1810 .

6d ${ }^{1} \mathrm{H}$ NMR (400 MHz, $\left.\mathrm{CDCl}_{3}\right) \delta 7.72\left(\mathrm{~d},{ }^{3} J=8.7 \mathrm{~Hz}, 1 \mathrm{H}\right), 7.32$ (s, $5 \mathrm{H}), 6.67$ (d, $\left.{ }^{3} J=8.7 \mathrm{~Hz}, 1 \mathrm{H}\right), 5.87$ (pt, $\left.J=5.5,2.0 \mathrm{~Hz}, 1 \mathrm{H}\right)$, 5.60 (dhpt, $J=11.2,1.7 \mathrm{~Hz}, 1 \mathrm{H}), 4.64-4.61(\mathrm{~m}, 2 \mathrm{H}), 3.56(\mathrm{~s}, 4 \mathrm{H})$, $2.65(\mathrm{~s}, 4 \mathrm{H}), 2.38(\mathrm{td}, J=11.5 \mathrm{~Hz}, 2 \mathrm{H}), 2.03(\mathrm{brd}, 2 \mathrm{H}), 1.80-1.68$ $(\mathrm{m}, 2 \mathrm{H}) .{ }^{13} \mathrm{C} \mathrm{NMR}\left(100 \mathrm{MHz}, \mathrm{CDCl}_{3}\right) \delta 191.5,165.4,156.6,137.8$, 129.2, 128.2 , 127.5, 127.1, 126.5, 125.9, 123.8, 117.3, 115.0, 78.2, 70.4, 63.1, 48.7, 34.3, 22.5. MS (EI) LRMS: $m / z$ 375, 189, 97, 91 (100\%), 71, 69, 57. HRMS (EI): $m / z$ calcd for $\mathrm{C}_{24} \mathrm{H}_{25} \mathrm{NO}_{3}$ is 375.1834; found: 375.1813 .

6e ${ }^{1} \mathrm{H}$ NMR (400 MHz, $\left.\mathrm{CDCl}_{3}\right) \delta 7.73\left(* 7.68, \mathrm{~d},{ }^{3} J=8.5 \mathrm{~Hz}\right.$, $1 \mathrm{H}), 7.31-7.26(\mathrm{~m}, 3 \mathrm{H}), 7.24-7.19(\mathrm{~m}, 2 \mathrm{H}), 6.67\left(* 6.61, \mathrm{~d},{ }^{3} \mathrm{~J}=\right.$ $8.7 \mathrm{~Hz}, 1 \mathrm{H}), 5.93\left\{\mathrm{pt},{ }^{3} J=5.5,2.0 \mathrm{~Hz},{ }^{*} 6.38\left(\mathrm{dt},{ }^{3} J=7.2,1.5 \mathrm{HZ}\right)\right.$, $1 \mathrm{H}\}, 5.57\{\mathrm{dhpt}, J=11.2,1.5 \mathrm{~Hz}, * 5.43$ (brs), $1 \mathrm{H}\}, 4.64-4.62$ $\left\{\mathrm{m}, * 4.30\left(\mathrm{t},{ }^{3} J=4.7 \mathrm{~Hz}\right), 2 \mathrm{H}\right\}, 3.61-3.60\{\mathrm{~m}, 2 \mathrm{H}, * 3.66(\mathrm{t}, J=$ $7.0 \mathrm{~Hz}, 2 \mathrm{H})\}, 2.85-2.77(\mathrm{~m}, 4 \mathrm{H}), 2.68(\mathrm{~s}, 2 \mathrm{H}) 2.66-2.63(\mathrm{~m}, 2 \mathrm{H})$, 2.52-2.40 (m, 2H), 2.10 (brd, 2H), 1.81-1.73 (m, 2H). ${ }^{13} \mathrm{C}$ NMR $\left(100 \mathrm{MHz}, \mathrm{CDCl}_{3}\right) \delta 191.4,165.4,156.5,140.2,131.0,128.6$, 128.4, 127.6, 126.4, 126.1, 126.0, 123.8, 119.7, 117.3, 115.0, 78.1, 70.4, 60.5, 49.0, 34.3, 33.7, 22.5. MS (EI) LRMS: $m / z$ 389, 360, 359 (100\%), 298, 110, 105, 91, 83, 71. HRMS (EI): $\mathrm{m} / \mathrm{z}$ calcd for $\mathrm{C}_{25} \mathrm{H}_{27} \mathrm{NO}_{3}$ is 389.1990; found: 389.1989 .

6f ${ }^{1} \mathrm{H}$ NMR (400 MHz, $\left.\mathrm{CDCl}_{3}\right) \delta 7.68\left(* 7.60, \mathrm{~d},{ }^{3} J=8.5 \mathrm{~Hz}\right.$, $1 \mathrm{H}), 6.65\left(* 6.57, \mathrm{~d},{ }^{3} J=8.7 \mathrm{~Hz}, 1 \mathrm{H}\right), 5.82\left\{\mathrm{pt},{ }^{3} J=5.7\right.$, $\left.2.0 \mathrm{~Hz},{ }^{*} 6.04,(\mathrm{dt}, J=12.0,4.2 \mathrm{~Hz}), 1 \mathrm{H}\right\}, 5.51\{\mathrm{dhpt}, J=11.2$, 
$1.5 \mathrm{~Hz}, * 5.38-5.33(\mathrm{~m}), 1 \mathrm{H}\}, 4.58-4.55(\mathrm{~m}, 2 \mathrm{H}), 4.37$ (dp, $J=$ 13.5, $2.0 \mathrm{~Hz}, 1 \mathrm{H}), 3.61$ (dp, $J=13.5,2.0 \mathrm{~Hz}, 1 \mathrm{H}), 3.52-3.51$ (m, $2 \mathrm{H}), 3.36(\mathrm{td}, 1 \mathrm{H}), 2.92(\mathrm{td}, 1 \mathrm{H}), 2.61(\mathrm{~s}, 2 \mathrm{H}), 2.04(\mathrm{~s}, 5 \mathrm{H}), 1.54$ (qd, 2H). ${ }^{13} \mathrm{C} \mathrm{NMR}\left(100 \mathrm{MHz}, \mathrm{CDCl}_{3}\right) \delta 190.6,169.0,165.6,156.0$, 131.6, 127.7, 126.1, 123.8, 117.1, 115.5, 114.2, 78.0, 70.4, 47.6, 42.0, 37.0, 34.4, 33.8, 22.4, 21.4. MS (EI) LRMS: $m / z, 312,214$, $189(100 \%), 160,96,91,82,55$. HRMS (EI): $\mathrm{m} / z$ calcd for $\mathrm{C}_{20} \mathrm{H}_{25} \mathrm{NO}_{3}$ is 327.1834: found: 327.1831 .

$6 \mathbf{g}^{1} \mathrm{H}$ NMR $\left(400 \mathrm{MHz}, \mathrm{CDCl}_{3}\right) \delta 7.69\left(* 7.73, \mathrm{~d},{ }^{3} J=8.7 \mathrm{~Hz}, 1 \mathrm{H}\right)$, $7.34(\mathrm{~s}, 5 \mathrm{H}), 6.63\{\mathrm{dt}, J=12.2,1.7 \mathrm{~Hz}\}, 6.64\left(* 6.71, \mathrm{~d},{ }^{3} J=8.7 \mathrm{~Hz}\right.$, $1 \mathrm{H}), 6.19\{\mathrm{dt}, J=12.2,4.5 \mathrm{~Hz}, * 5.92(\mathrm{pt}, J=5.5,2.0 \mathrm{~Hz}), 1 \mathrm{H}\}, 5.59$ $(\mathrm{t}, J=4.7 \mathrm{~Hz}, 2 \mathrm{H}), 4.57$ (s, 2H), 4.48 (brs, 1H), $3.53(\mathrm{~s}, 2 \mathrm{H}), 3.22$ (brd, 2H), 2.65 (s, 2H) 1.86-1.73 (m, 2H), 2.01-1.85 (m, 1H). ${ }^{13} \mathrm{C}$ NMR $\left(100 \mathrm{MHz}, \mathrm{CDCl}_{3}\right) \delta 190.2,170.5,166.0,157.7,142.7,135.6$, 131.7, 129.8, 128.5, 126.9, 126.1, 119.2, 116.0, 115.7, 114.2, 78.6, 70.4, 47.4, 34.1. MS (EI) LRMS: $m / z$ 389, 229, 177, 149, 105 (100\%). HRMS (EI): $m / z$ calcd for $\mathrm{C}_{24} \mathrm{H}_{23} \mathrm{NO}_{4}$; found: 389.1600 .

6h ${ }^{1} \mathrm{H}$ NMR (400 MHz, $\left.\mathrm{CDCl}_{3}\right) \delta=7.74\left(\mathrm{~d},{ }^{3} J=8.5 \mathrm{~Hz}, 1 \mathrm{H}\right)$, 7.37-7.35 (m, 5H), 6.71 (d, $\left.{ }^{3} J=8.7 \mathrm{~Hz}, 1 \mathrm{H}\right), 5.91$ (pt, ${ }^{3} J=5.5$, $2.0 \mathrm{~Hz}, 1 \mathrm{H}$ ), 5.58 (dhpt, $J=11.2,1.7 \mathrm{~Hz}, 1 \mathrm{H}), 5.13$ (s, 2H), 4.644.62 (m, 2H), 4.02 (brs, 2H), 3.59 (m, 2H), 3.19 (brs, 2H), 2.67 (s, 2H), 2.06 (brs, 2H), 1.64-1.56 (m, 2H). ${ }^{13} \mathrm{C} \mathrm{NMR} \mathrm{(100} \mathrm{MHz,} \mathrm{CDCl}_{3}$ ) $\delta$ 190.7, 165.6, 156.1, 155.2, 136.6, 128.5, 128.1, 127.9, 127.6, 126.2, 126.1, 123.7, 117.2, 115.4, 78.0, 70.4, 67.2, 47.7, 39.5, 33.9, 22.4. MS (EI) LRMS: $m / z$ 419, 328 (100\%), 284, 187, 91. HRMS (EI): $m / z$ calcd for $\mathrm{C}_{25} \mathrm{H}_{25} \mathrm{NO}_{5}$ is 419.1732 ; found: 419.1750 .

$6 \mathbf{i}^{1} \mathrm{H}$ NMR $\left(400 \mathrm{MHz}, \mathrm{CDCl}_{3}\right) \delta 7.74\left(* 7.69, \mathrm{~d},{ }^{3} J=8.5 \mathrm{~Hz}\right.$, $1 \mathrm{H}), 6.71\left(* 6.53, \mathrm{~d},{ }^{3} \mathrm{~J}=8.7 \mathrm{~Hz}, 1 \mathrm{H}\right), 5.92\left\{\mathrm{pt},{ }^{3} J=5.5\right.$, $2.0 \mathrm{~Hz}, * 6.08(\mathrm{dt}, J=12.2,4.5 \mathrm{~Hz}), * 6.37(\mathrm{dt}, J=7.2,2.0 \mathrm{~Hz}), 1 \mathrm{H}\}$, $5.60\{\mathrm{dhpt}, J=11.2,1.7 \mathrm{~Hz}, 1 \mathrm{H}\}, 4.63\{(\mathrm{dt}, J=7.5,4.2 \mathrm{~Hz}), * 4.96-$ $4.83(\mathrm{~m}), 2 \mathrm{H}\}, 4.14$ (q, $J=7.2 \mathrm{~Hz}, 2 \mathrm{H}), 3.97$ (brs, $2 \mathrm{H}), 3.58-3.50$ $\left\{\mathrm{m},{ }^{*} 3.68-3.63(\mathrm{~m}), 2 \mathrm{H}\right\}, 3.27-3.10(\mathrm{~m}, 2 \mathrm{H}), 2.68(\mathrm{~s}, 2 \mathrm{H}), 2.07$ (brd, 2H), 1.62-1.57 (m, 2H), 1.25 (t, $J=7.2 \mathrm{~Hz}, 3 \mathrm{H}) .{ }^{13} \mathrm{C}$ NMR $\left(100 \mathrm{MHz}, \mathrm{CDCl}_{3}\right) \delta 189.8,164.5,155.2,154.4,126.6,125.2$, 125.1, 116.2, 114.3, 122.7, 77.1, 69.4, 60.5, 46.7, 38.3, 32.9, 21.4, 13.6. MS (EI) LRMS: $m / z$ 357, 342, 189 (100\%), 160. HRMS (EI): $m / z$ calcd for $\mathrm{C}_{20} \mathrm{H}_{23} \mathrm{NO}_{5}$ is 357.1576; found 357.1570.

6j ${ }^{1} \mathrm{H}$ NMR $\left(400 \mathrm{MHz}, \mathrm{CDCl}_{3}\right) \delta 7.69\left(* 7.61, \mathrm{~d},{ }^{3} J=8.5 \mathrm{~Hz}\right.$, $1 \mathrm{H}), 6.64\left({ }^{*} 6.54, \mathrm{~d},{ }^{3} J=8.7 \mathrm{~Hz}, 1 \mathrm{H}\right), 5.83\left\{\mathrm{pt},{ }^{3} J=5.7,2.0 \mathrm{~Hz}\right.$, *6.04, (dt, $J=12.0,4.2 \mathrm{~Hz}), 1 \mathrm{H}\}, 5.50(\mathrm{dhpt}, J=11.2,1.5 \mathrm{~Hz}, 1 \mathrm{H})$, 4.57-4.55 (m, 2H), 3.89 (brs, 2H), 3.52-3.50 (m, 2H), 3.06 (brs, 2H), 2.60 (s, 2H), 1.95 (brd, 2H), 1.64-1.52 (m, 2H), 1.39 (s, 9H). ${ }^{13} \mathrm{C}$ NMR $\left(100 \mathrm{MHz}, \mathrm{CDCl}_{3}\right) \delta 189.9,164.5,155.2,153.6,126.6$, 125.3, 125.0, 122.7, 116.2, 114.3, 78.8, 77.1, 69.4, 46.7, 33.0, 27.4, 21.4. MS (EI) LRMS: $m / z$ 385, 330, 329 (100\%) 314, 189, 96. HRMS (EI): $m / z$ calcd for $\mathrm{C}_{22} \mathrm{H}_{27} \mathrm{NO}_{5}$ : 385.1889 ; found: 385.1900 .

\section{Conflicts of interest}

There are no conflicts to declare.

\section{Acknowledgements}

We thank the Department of Science and Technology (DST), India for supporting this research, under the scheme "Disha Programme for Women in Science". This project was also funded initially by KIRAN-Societal Research Fellowship (SoRF), DST.

\section{References}

1 L. F. Tietze, H. P. Bell and S. Chandrasekhar, Angew. Chem., 2003, 42, 3996-4028.

2 R. Romagnoli, P. Baraldi, M. K. Salvador, M. E. Camacho, J. Balzarini, J. Bermejo and F. Estévez, Eur. J. Med. Chem., 2013, 63, 544-557.

3 E. M. Guantai, K. Ncokazi, T. J. Egan, J. Gut, P. J. Rosenthal, P. J. Smith and K. Chibale, Bioorg. Med. Chem. Lett., 2010, 18, 8243-8256.

4 B. Meunier, Acc. Chem. Res., 2008, 41, 69-77.

5 Y. Wu, M. Hu, L. Yang, X. Li, J. Bian, F. Jiang, H. Sun, Q. You and X. Zhang, Bioorg. Med. Chem. Lett., 2015, 25, 2584-2588.

6 L. He, Y. Ling, L. Fu, D. Yin, X. Wang and Y. Zhang, Bioorg. Med. Chem. Lett., 2012, 22, 289-292.

7 X. Zhang, X. Li, H. Sun, Z. Jiang, L. Tao, Y. Gao, Q. Guo and Q. You, Org. Biomol. Chem., 2012, 10, 3288-3299.

8 K. M. Elbel, G. Guizzunti, M. A. Theodoraki, J. Xu, A. Batova, M. Dakanali and E. A. Theodorakis, Org. Biomol. Chem., 2013, 11, 3341-3348.

9 X. Zhang, X. Li, H. Sun, X. Wang, L. Zhao, Y. Gao, X. Liu, S. Zhang, Y. Wang, Y. Yang, S. Zeng, Q. Guo and Q. You, J. Med. Chem., 2013, 56, 276-292.

10 L. Feng, M. M. Maddox, M. Z. Alam, L. S. Tsutsumi, G. Narula, D. F. Bruhn, X. Wu, S. Sandhaus, R. B. Lee, C. J. Simmons, Y.-C. Tse-Dinh, J. G. Hurdle, R. E. Lee and D. Sun, J. Med. Chem., 2014, 57, 8398-8420.

11 H. G. Bonacorso, W. C. Rosa, S. M. Oliveira, I. Brusco, E. S. Brum, M. B. Rodrigues, C. P. Frizzo and N. Zanatta, Bioorg. Med. Chem. Lett., 2017, 27, 1551-1556.

12 U. M. Battisti, S. Corrado, C. Sorbi, A. Cornia, A. Tait, D. Malfacini, M. C. Cerlesi, G. Calo and L. Brasili, Med. Chem. Commun., 2014, 5, 973-983.

13 M. Mujahid, R. G. Gonnade, P. Yogeeswari, D. Sriram and M. Muthukrishnan, Bioorg. Med. Chem. Lett., 2013, 23, 1416-1419.

14 L. Zhang, V. Dewan and H. Yin, J. Med. Chem., 2017, 60, 5029-5044.

15 D. Ashok, E. V. L. Madhuri, M. Sarasija, S. Sree Kanth, M. Vijjulatha, M. D. Alaparthi and S. R. Sagurthi, RSC Adv., 2017, 7, 25710-25724.

16 K. Sambasivarao, M. Kalyaneswar, T. Arti and M. M. Shaikh, Chem.-Eur. J., 2006, 12, 8024-8038.

17 L. Yet, Chem. Rev., 2000, 100, 2963-3008.

18 G. A. Molander, Acc. Chem. Res., 1998, 31, 603-609.

19 A. K. Chatterjee, J. P. Morgan, M. Scholl and R. H. Grubbs, J. Am. Chem. Soc., 2000, 122, 3783-3784.

20 V. Declerck, H. Allouchi, J. Martinez and F. Lamaty, J. Org. Chem., 2007, 72, 1518-1521.

21 M. Arisawa, A. Nishida and M. Nakagawa, J. Organomet. Chem., 2006, 691, 5109-5121.

22 J. Burnley, Z. J. Wang, W. R. Jackson and A. J. Robinson, J. Org. Chem., 2017, 82, 8497-8505.

23 B. J. Ireland, B. T. Dobigny and D. E. Fogg, ACS Catal., 2015, 5, 4690-4698. 